\title{
The role of molecular gas in galaxy transition in compact groups ${ }^{\star}$
}

\author{
U. Lisenfeld ${ }^{1}$, K. Alatalo ${ }^{2}$, C. Zucker ${ }^{3}$, P. N. Appleton ${ }^{4}$, S. Gallagher ${ }^{5,6}$, P. Guillard ${ }^{7,8}$, and K. Johnson ${ }^{9}$ \\ ${ }^{1}$ Departamento de Física Teórica y del Cosmos, Universidad de Granada, Spain and Instituto Carlos I de Física Téorica y \\ Computacional, Facultad de Ciencias, 18071 Granada, Spain \\ e-mail: ute@ugr.es \\ 2 Observatories of the Carnegie Institution of Washington, 813 Santa Barbara Street, Pasadena, CA 91101, USA \\ 3 Harvard-Smithsonian Center for Astrophysics, Cambridge, MA 02138, USA \\ 4 NASA Herschel Science Center, IPAC, Caltech, Pasadena, CA 91125, USA \\ 5 Department of Physics and Astronomy, University of Western Ontario, London, ON N6A 3K7, Canada \\ ${ }^{6}$ Centre for Planetary and Space Exploration, University of Western Ontario, London, ON N6A 3K7, Canada \\ 7 Institut d'Astrophysique de Paris, CNRS, UMR 7095, 98bis boulevard Arago, 75014 Paris, France \\ 8 Sorbonne Universités, UPMC Université Paris 06, 4 place Jussieu, 75005 Paris, France \\ 9 Department of Astronomy, University of Virginia, PO Box 400325, Charlottesville, VA 22904-4325, USA
}

Received 30 March 2017 / Accepted 2 August 2017

\begin{abstract}
Compact groups (CGs) provide an environment in which interactions between galaxies and with the intra-group medium enable and accelerate galaxy transitions from actively star forming to quiescent. Galaxies in transition from active to quiescent can be selected, by their infrared (IR) colors, as canyon or infrared transition zone (IRTZ) galaxies. We used a sample of CG galaxies with IR data from the Wide Field Infrared Survey Explorer (WISE) allowing us to calculate the stellar mass and star formation rate (SFR) for each galaxy. Furthermore, we present new $\mathrm{CO}(1-0)$ data for 27 galaxies and collect data from the literature to calculate the molecular gas mass for a total sample of 130 galaxies. This data set allows us to study the difference in the molecular gas fraction $\left(M_{\text {mol }} / M_{*}\right)$ and star formation efficiency $\left(S F E=S F R / M_{\text {mol }}\right.$ ) between active, quiescent, and transitioning (i.e., canyon and IRTZ) galaxies. We find that transitioning galaxies have a mean molecular gas fraction and a mean SFE that are significantly lower than those of actively star-forming galaxies. The molecular gas fraction is higher than that of quiescent galaxies, whereas the SFE is similar. These results indicate that the transition from actively star-forming to quiescent in CG galaxies goes along with a loss of molecular gas, possibly due to tidal forces exerted from the neighboring galaxies or a decrease in the gas density. In addition, the remaining molecular gas loses its ability to form stars efficiently, possibly owing to turbulence perturbing the gas, as seen in other, well-studied examples such as Stephan's Quintet and HCG 57. Thus, the amount and properties of molecular gas play a crucial role in the environmentally driven transition of galaxies from actively star forming to quiescent.
\end{abstract}

Key words. ISM: molecules - galaxies: interactions - galaxies: evolution - galaxies: ISM - galaxies: star formation galaxies: groups: general

\section{Introduction}

Galaxies show a bimodal distribution in optical color space, most of which are either in a blue cloud or a red sequence (e.g., Baldry et al. 2004). The dearth of galaxies in the green valley, in between the red and blue galaxies, has been interpreted as a rapid transition between the two phases (Bell et al. 2004; Faber et al. 2007). The mechanisms responsible for this transition are not clear and there are most likely different causes as seen, for example, in the analysis of Schawinski et al. (2014). These authors demonstrated that the green valley does not consist of a homogeneous group of galaxies, but is composed of fast-evolving earlytype galaxies and secularly evolving late-type galaxies. This shows that optical colors alone are not enough to study galaxy transitions.

Infrared (IR) colors of galaxies can give complementary information. Based on WISE colors, Alatalo et al. (2014b) found a prominent bifurcation between late-type and early-type galaxies in the WISE color range of $0.8 \lesssim W 2-W 3 \lesssim 2.4$, which

* Full Table 2 is only available at the CDS via anonymous ftp to cdsarc.u-strasbg.fr (130.79.128.5) or via

http://cdsarc.u-strasbg.fr/viz-bin/qcat?J/A+A/607/A110 they labeled the infrared transition zone (IRTZ). Interestingly, the IRTZ does not coincide with the optical green valley. Instead, galaxies in the IRTZ tend to have mostly red optical colors and are predominantly early-type galaxies. This indicates that galaxies seem to pass the optical green valley first, and later the IRTZ, possibly by completely shedding their interstellar medium (ISM; Alatalo et al. 2014b). A similar result was found by Walker et al. (2013) for galaxies in compact groups (CG).

In an analysis of Spitzer Space Observatory IRAC colors, Johnson et al. (2007) found an IR gap, i.e., a seemingly underpopulated region, in Hickson compact groups (HCGs), separating actively star-forming galaxies from galaxies dominated by an evolved stellar population. The low density of galaxies in this gap suggests that the evolutionary phase corresponding to the gap is a very short transition. The existence of an underpopulated region was confirmed in larger samples (Walker et al. 2010) but confined to a smaller region in mid-IR color space (Walker et al. 2012). With star formation rates (SFR) and stellar masses measured from UV and near- and mid-IR photometry, Tzanavaris et al. (2010) identified specific star formation rate (sSFR) as the physical driver for location in mid-IR color space. 
Zucker et al. (2016) expanded these studies, based on a limited amount of Spitzer data, using WISE data. They measured the fluxes in all four WISE bands for a sample of 652 galaxies in 163 CGs and classified these galaxies, using colors, into mid-IR active (meaning actively star-forming), mid-IR quiescent, and mid-IR canyon galaxies ${ }^{1}$. Their method consisted in plotting the galaxies in a unique region of WISE color-color space $\log (W 3-W 2)$ vs. $\log (W 4-W 1)$ to separate galaxies dominated by polyaromatic hydrocarbon (PAH) emission from those dominated by stellar light. Zucker et al. then applied 2D isodensity contours to identify canyon galaxies occupying the underdense region between the active and quiescent populations. These authors showed that this classification is compatible with the previous classification based on Spitzer data, allowing the enlargement of the sample by a factor of three owing to the complete sky coverage of the WISE observations. Galaxies classified as belonging to the canyon between active and quiescent also exhibit a sSFR between active and quiescent galaxies, implying that this population represents a transition phase between the two in which the star formation (SF) is quenched.

Additional indications that something unusual is going on in these galaxies was found by Cluver et al. (2013) who observed a sample of Hickson compact groups (HCGs) with intermediate HI deficiencies with the Spitzer IRS spectrograph. These authors identified a number of galaxies with enhanced warm $\mathrm{H}_{2}$ emission that is well above the level expected from SF and indicative of shock excitation, classifying these galaxies as so-called molecular hydrogen emission galaxies (MOHEGs; Ogle et al. 2010). Interestingly, most of these galaxies have IRAC colors in the range of the previously found gap (Johnson et al. 2007; Walker et al. 2010). Furthermore, based on their extinction-corrected optical colors, these galaxies fall predominantly in the optical green valley between blue, star-forming galaxies and red-sequence objects. Cluver et al. (2013) conclude that the increased warm $\mathrm{H}_{2}$ emission is most likely due to shock excitation.

The cause of the transition between active and quiescent is still unclear. The transition goes in parallel with a decrease in the SFR, yet it is unclear what exactly shuts the SF down. In order to shed further light on this question, we need to quantify the SFR and also measure and characterize the molecular gas content that is available. Previous surveys of the molecular gas (Leon et al. 1998; Verdes-Montenegro et al. 1998; Martinez-Badenes et al. 2012) found no deficiency of the molecular gas compared to field galaxies. Lisenfeld et al. (2014) measured the molecular gas content in a small sample of 31 HCG galaxies; they classified most of these with available data for the warm $\mathrm{H}_{2}$ emission and classified 14 as MOHEGs. Even though there were some objects with a very low SFE, there was no clear relation to the intensity of the warm $\mathrm{H}_{2}$ emission; instead, on average the SFE in MOHEG galaxies was the same as in non-MOHEG galaxies. On the other hand, the $M_{\mathrm{H}_{2}} / L_{\mathrm{K}}$ ratio was lower for MOHEG galaxies, indicating a decrease in the molecular gas content. Some of the galaxies (mostly MOHEGs) showed very broad lines $\left(\sim 1000 \mathrm{~km} \mathrm{~s}^{-1}\right)$ and irregular line shapes that are indicative of a perturbation of the molecular gas. The presence of kinematically disturbed molecular gas was confirmed with CARMA observations in HCG 57 (Alatalo et al. 2014a) and later in a sample of 14 HCGs (Alatalo et al. 2015), in which a considerable SF suppression was found in some objects, especially in those galaxies that were in a more advanced stage of transition based on their optical and IR colors.

\footnotetext{
1 In the most parts of this paper, and following Zucker et al. (2016), we call these groups simply active, quiescent and canyon galaxies.
}

The goal of the present paper is to clarify the role that molecular gas plays in the decrease of SF that goes along with the transition from active to quiescent. We approach this question based on a sample of CG galaxies. Because of their low velocity dispersions and close proximity to other group members, these galaxies are strongly affected by interactions with their companions and the intra-group medium. The effects are clearly visible as evidenced by a large percentage of the atomic hydrogen (HI) that is outside the galaxies in some groups (e.g., Verdes-Montenegro et al. 2001) and by the morphological dominance of S0 galaxies. We use the sample of Zucker et al. (2016) to study the relation between molecular gas, SF, and stellar mass as a function of IR color. The sample and the CO data presented in this paper allow us to answer the fundamental question of whether there are differences in the mean molecular gas fraction and star formation efficiency (SFE; defined as the SFR per molecular gas mass) between active, quiescent, and transitioning galaxies. The classification into these groups is based on their mid-IR colors. We consider two different groups of transitioning galaxies: canyon galaxies as defined by Zucker et al. (2016) and IRTZ galaxies as defined by Alatalo et al. (2014b).

\section{Sample and data}

Our sample is based on the catalog of Zucker et al. (2016), which presents WISE data for 652 galaxies in 163 compact groups, of which 428 galaxies have reliable photometry $(S / N>2$ in all bands). The groups are composed of 93 HCGs, identified in a systematic visual search by Hickson (1982) and later cleaned up with the availability of velocity data (Hickson et al. 1992). In addition, the sample includes 70 groups from the Redshift Survey Compact Group catalog (RSCG; Barton et al. 1996) whose galaxies were chosen from a complete, magnitude-limited redshift survey via a friends-of-friends algorithm. Zucker et al. (2016) excluded discordant HCG groups and galaxies, checked the two samples for overlap, and excluded RSCGs that were embedded in clusters. In addition to this and following Barton et al. (1996), we excluded very close-by systems $\left(c z \leq 2300 \mathrm{~km} \mathrm{~s}^{-1}\right)$, whose environment cannot be analyzed reliably due to their large angular sizes. We also implemented a stricter signal-to-noise cut than in Zucker et al. (2016) and excluded any galaxies with $S / N<2.5$ in lieu of the $S / N<2$ cut they used. In all cases, the $W 4$ band was the limiting factor for this criterion; i.e., if a galaxy was detected in $W 4$, it was detected in the other bands as well. Applying both the redshift and signal-to-noise cuts, we narrow our potential sample down to 294 of 428 galaxies used in the Zucker et al. (2016) analysis.

We searched the literature for all existing $\mathrm{CO}$ data for this Zucker et al. (2016) subsample (294 galaxies) and obtained CO measurements for $102 \mathrm{HCG}$ galaxies (Verdes-Montenegro et al. 1998; Leon et al. 1998; Martinez-Badenes et al. 2012; Lisenfeld et al. 2014) and for two RSCG galaxies (Mirabel et al. 1990; Wiklind et al. 1995, NGC 232 and NGC 2831) ${ }^{2}$. The observations were carried out with the Institut de Radioastronomie Millimetrique (IRAM) $30 \mathrm{~m}$ telescope, Five College Radio Telescope, Swedish-ESO Submillimetre Telescope (SEST), and Kitt

Three galaxies that were all classified as active by Zucker et al. (2016), with available CO and WISE data, were not included in the present study. This is because of possible contamination of the mid-IR emission by an AGN (HCG 56b and HCG 96a; Cluver et al. 2013) and an unusually high SFE in the merging object HCG 31 ac that is possibly due to an intergalactic starburst, making it atypical for our study (see Lisenfeld et al. 2014, for a discussion). 
Peak Radio Telescope with single pointings at the central position for most cases. To supplement the $\mathrm{CO}$ data for these 104 galaxies from the literature, as part of this study we observed the redshifted $\mathrm{CO}(1-0)$ line for an additional 27 galaxies. The details of these observations are described in Sect. 2.1 below. One of the objects, HCG 54a, was later excluded from the statistical analysis because of its low recession velocity, but we present the $\mathrm{CO}$ data here for completeness. In total, our final sample includes CO data for 130 galaxies (104 from the literature and 26 obtained herein, of which 89 are detections), which constitutes about one-third of the reliable photometric sample of Zucker et al. (2016).

Our selection of galaxies from the sample of Zucker et al. (2016) was driven by the availability of CO data. Of the 294 galaxies from Zucker et al. (2016) with reliable mid-IR photometry $(S / N>2.5$ in all four WISE bands), only 130 had available $\mathrm{CO}$ data. While we are not affected by any obvious biases because each $\mathrm{CO}$ study from the literature used different selection criteria, we nevertheless need to check that our results using this 130 galaxy subsample are robust. We carried out this test by creating an additional subsample that only contained galaxies in those groups with complete $\mathrm{CO}$ coverage, yielding 89 of 130 galaxies. Explicitly, we went through the Zucker et al. (2016) sample group by group and determined whether all galaxies in that group with reliable photometry $(S / N>2.5)$ also had CO data. If they did, all reliable photometry galaxies in that group were included in the bias test. Otherwise all galaxies were excluded. This allowed the compact group environment to remain intact with respect to the Zucker et al. (2016) sample, by avoiding the inclusion of only some galaxies in a group that might have been preferentially selected for CO follow-up observations. In this way, these 89 of 130 galaxies were selected based on the $W 4$ flux rather than $\mathrm{CO}$. We carried out the entire analysis for this smaller 89 galaxy subsample and obtained entirely consistent results, indicating that the availability of $\mathrm{CO}$ data did not affect our conclusions. The details are presented in Appendix A.

\subsection{CO observations}

We observed an additional 27 galaxies in CGs between January and April 2017 with the IRAM $30 \mathrm{~m}$ telescope on Pico Veleta. We selected the sources, based on their WISE colors, as preferentially canyon or IRTZ objects. We observed the redshifted ${ }^{12} \mathrm{CO}(1-0)$ line with the dual polarization receiver EMIR in combination with the autocorrelator FTS at a frequency resolution of $0.195 \mathrm{MHz}$ (providing a velocity resolution of $\sim 0.5 \mathrm{~km} \mathrm{~s}^{-1}$ at $\mathrm{CO}(1-0)$ ) and with the autocorrelator WILMA with a frequency resolution of $2 \mathrm{MHz}$ (providing a velocity resolution of $\sim 5 \mathrm{~km} \mathrm{~s}^{-1}$ at $\left.\mathrm{CO}(1-0)\right)$. The observations were carried out in wobbler switching mode with a wobbler throw between $40^{\prime \prime}$ and $140^{\prime \prime}$ in azimuthal direction. The wobbler throw was chosen individually in order to ensure that the off position was away from neighboring galaxies.

The broad bandwidth of the receiver $(16 \mathrm{GHz})$ and backends (8 GHz for the FTS and $4 \mathrm{GHz}$ for WILMA) enabled grouping of observations of galaxies with similar redshifts. The central sky frequencies, taking into account the redshift of the objects, ranged between 108 and $112 \mathrm{GHz}$. Each object was observed until it was detected with a $\mathrm{S} / \mathrm{N}$ ratio of $\sim 5$ or until the rootmean-square (rms) noise was below $\sim 3 \mathrm{mK}\left(T_{\mathrm{A}}^{*}\right.$ ) for a velocity resolution of $20 \mathrm{~km} \mathrm{~s}^{-1}$. The integration times per object ranged from 30 to $130 \mathrm{~min}$. Pointing was monitored on nearby quasars every 60-90 min. During the observation period, the weather conditions were generally good with a pointing accuracy better than $3^{\prime \prime}$. The mean system temperature for the observations was $160 \mathrm{~K}$ on the $T_{\mathrm{A}}^{*}$ scale. At $115 \mathrm{GHz}$, the IRAM forward efficiency, $F_{\text {eff }}$, is 0.95 and the beam efficiency, $B_{\text {eff }}$, is 0.77 . The half-power beam size is 22.3" (for $110 \mathrm{GHz}$ ). All CO spectra and luminosities are presented on the main beam temperature scale $\left(T_{\mathrm{mb}}\right)$, which is defined as $T_{\mathrm{mb}}=\left(F_{\mathrm{eff}} / B_{\mathrm{eff}}\right) \times T_{\mathrm{A}}^{*}$.

The data were reduced in the standard way via the CLASS software in the GILDAS package ${ }^{3}$. We first discarded poor scans and then subtracted a constant or linear baseline. We then averaged the spectra and smoothed them to resolutions between 20 and $40 \mathrm{~km} \mathrm{~s}^{-1}$ to increase the signal-to-noise $(\mathrm{S} / \mathrm{N})$ ratio.

We present the detected spectra in Fig. A1. For each spectrum, we determined visually the zero-level line widths, if detected. The velocity integrated spectra were calculated by summing the individual channels in between these limits. For non-detections we set an upper limit as

$I_{\mathrm{CO}}<3 \times \mathrm{rms} \times \sqrt{\delta V \Delta V}$,

where $\delta V$ is the channel width, $\Delta V$ the zero-level line width, and rms the root mean square noise. For the non-detections, we assumed a linewidth of $\Delta V=300 \mathrm{~km} \mathrm{~s}^{-1}$. We treated tentative detections, with a $\mathrm{S} / \mathrm{N}$ ratio between $4-5$, as upper limits in the statistical analysis. The results of our $\mathrm{CO}(1-0)$ observations are listed in Table 1.

\subsection{Molecular gas mass}

We calculated the molecular gas mass within the pointing, $M_{\text {mol, },}$, from the $\mathrm{CO}(1-0)$ emission with the following equation:

$M_{\mathrm{mol}, 0}\left[M_{\odot}\right]=102 \times D^{2} I_{\mathrm{CO}(1-0)} \Omega$.

Here, $\Omega$ is the area covered by the observations in $\operatorname{arcsec}^{2}$ (i.e., $\Omega=1.13 \theta^{2}$ for a single pointing with a Gaussian beam where $\theta$ is the HPBW), $D$ is the distance in $\mathrm{Mpc}$, and $I_{\mathrm{CO}(1-0)}$ is the velocity integrated line intensity in $\mathrm{K} \mathrm{km} \mathrm{s}^{-1}$. This equation assumes a CO-to- $\mathrm{H}_{2}$ conversion factor $X=N_{\mathrm{H}_{2}} / I_{\mathrm{CO}}=2 \times 10^{20} \mathrm{~cm}^{-2}$ $\left(\mathrm{K} \mathrm{km} \mathrm{s}^{-1}\right)^{-1}$ (Bolatto et al. 2013) and includes a factor of 1.36 to account for helium and other heavy metals.

The CO data in our sample were obtained with different telescopes and different HPBWs. Furthermore, only the central positions were observed. Therefore, we need to apply a correction for emission outside the beam to derive the total molecular gas mass, which is necessary to be able to compare the data from different telescopes. We carried out this aperture correction in the same way as described in Lisenfeld et al. (2011), assuming an exponential distribution of the $\mathrm{CO}$ emission,

$I_{\mathrm{CO}}(r)=I_{0} \cdot \exp \left(r / r_{\mathrm{e}}\right)$.

We adopted a scale length of $r_{\mathrm{e}}=0.2 \times r_{25}$, where $r_{25}$ is the major optical isophotal radius at $25 \mathrm{mag} \operatorname{arcsec}^{-2}$, following Lisenfeld et al. (2011), who derived this scale length from different studies (Nishiyama et al. 2001; Regan et al. 2001; Leroy et al. 2008) and from their own CO data. These studies are based on spiral galaxies, however, the analysis of the spatial extent of CO in early-type galaxies in the $\operatorname{ALTAS}^{3 \mathrm{D}}$ survey showed that the relative $\mathrm{CO}$ extent, normalized to $r_{25}$, of early-type and spiral galaxies is the same (Davis et al. 2013).

http://www.iram.fr/IRAMFR/GILDAS 
Table 1. Integrated CO intensities.

\begin{tabular}{|c|c|c|c|}
\hline Galaxy & $\begin{array}{l}\mathrm{rms}^{a} \\
{[\mathrm{mK}]}\end{array}$ & $\begin{array}{c}I_{\mathrm{CO}(1-0)} \\
{\left[\mathrm{K} \mathrm{km} \mathrm{s}^{-1}\right]}\end{array}$ & $\begin{array}{c}\Delta V_{\mathrm{CO}(1-0)}{ }^{b} \\
{\left[\mathrm{~km} \mathrm{~s}^{-1}\right]}\end{array}$ \\
\hline HCG 01b & 1.58 & $1.41 \pm 0.15$ & 410 \\
\hline HCG 05a & 1.51 & $5.73 \pm 0.12$ & 275 \\
\hline HCG $19 \mathrm{c}$ & 3.24 & $<0.77$ & $300^{c}$ \\
\hline HCG 28a & 1.97 & $2.16 \pm 0.22$ & 570 \\
\hline HCG 32d & 1.27 & $0.83 \pm 0.14$ & 590 \\
\hline HCG 39a & 1.16 & $0.97 \pm 0.15$ & 800 \\
\hline HCG 45a & 1.08 & $1.98 \pm 0.17$ & 1120 \\
\hline HCG 45b & 1.08 & $0.62 \pm 0.14^{*}$ & 800 \\
\hline HCG 46b & 1.35 & $0.91 \pm 0.12$ & 370 \\
\hline HCG 47c & 1.89 & $2.47 \pm 0.18$ & 434 \\
\hline HCG 51b & 2.90 & $3.58 \pm 0.27$ & 415 \\
\hline HCG 52a & 1.32 & $0.79 \pm 0.14$ & 536 \\
\hline $\mathrm{HCG} 54 \mathrm{a}^{d}$ & 3.37 & $<0.80$ & 300 \\
\hline HCG 57b & 1.87 & $1.37 \pm 0.22$ & 634 \\
\hline HCG 57e & 1.85 & $1.22 \pm 0.19$ & 489 \\
\hline HCG 60b & 0.93 & $1.09 \pm 0.12$ & 715 \\
\hline HCG 64a & 2.44 & $1.71 \pm 0.23$ & 423 \\
\hline HCG 70a & 2.12 & $<0.51$ & $300^{c}$ \\
\hline HCG 70b & 2.45 & $2.69 \pm 0.27$ & 564 \\
\hline HCG 70c & 1.55 & $1.04 \pm 0.14$ & 375 \\
\hline HCG 71a & 2.36 & $6.71 \pm 0.24$ & 470 \\
\hline HCG 75c & 1.28 & $0.54 \pm 0.12 *$ & 389 \\
\hline NGC 0070 & 3.53 & $<0.85$ & $300^{c}$ \\
\hline NGC 4410NED01 & 3.09 & $5.11 \pm 0.35$ & 604 \\
\hline NGC 4614 & 1.94 & $1.56 \pm 0.15$ & 295 \\
\hline NGC 6961 & 2.50 & $<0.60$ & 300 \\
\hline $\mathrm{MCG}+02-36-018$ & 1.75 & $0.73 \pm 0.11$ & 200 \\
\hline
\end{tabular}

Notes. ${ }^{(a)}$ Root-mean-square noise level at a velocity resolution of $\sim 21 \mathrm{~km} \mathrm{~s}^{-1}$. ${ }^{(b)}$ Zero-level line width. The uncertainty is roughly given by the velocity resolution. ${ }^{(c)}$ Assumed linewidth for the non-detections. (d) HCG 54a was excluded from the subsequent statistical analysis because of its low recession velocity. ${ }^{(*)}$ Tentative detection ( $\mathrm{S} / \mathrm{N}$ between 4 and 5).

We used this distribution to calculate the expected $\mathrm{CO}$ emission from the entire disk, taking the galaxy inclination into account (see Lisenfeld et al. 2011, for more details). We obtained both the isophotal radius (corrected for galactic extinction and inclination) and the inclination from the Hyperleda ${ }^{4}$ database (Makarov et al. 2014).

The resulting aperture correction factor, $f_{\text {aper }}$, defined as the ratio between the molecular gas mass observed in the central pointing, $M_{\mathrm{mol}, 0}$ and the molecular gas mass extrapolated to the entire disk, $M_{\text {mol }}$, lies between 1.0 and 5.3 with a mean value of 1.7. The values for the molecular gas mass in the central pointing and the extrapolated molecular gas mass are listed in Table 2.

The empirical aperture correction could have potentially introduced biases in our analysis. We carried out two bias tests, described in detail in Appendix C, even though the small mean value of $f_{\text {aper }}(1.7)$, which is considerably less than the differences in SFE and $M_{\mathrm{mol}} / M_{*}$ found between the galaxy groups (factors $>3$ ), makes a strong bias unlikely. Firstly, we compared the distribution of $f_{\text {aper }}$ for the different galaxy groups studied in this analysis (active, quiescent, canyon, and IRTZ galaxies) and found that there is no significant difference. Secondly, we carried out our entire analysis for a subsample of galaxies with a small

\footnotetext{
4 http://leda.univ-lyon $1 . \mathrm{fr} /$
}

aperture correction $\left(f_{\text {aper }}<1.6\right)$. We found that the results are entirely consistent with the analysis of the full sample. Thus, we conclude that the application of the aperture correction did not bias our results.

\subsection{Star formation rate}

The mid-IR emission is a good tracer for the SFR. We used the WISE data in band 4 ( $W 4, \lambda=22 \mu \mathrm{m}$ ) from Zucker et al. (2016) to calculate the SFR. We adopted the prescription of Lee et al. (2013) who calibrated this band as a SFR tracer for a sample of $\sim 100000$ galaxies retrieved from the SDSS, based on a comparison with the extinction corrected $\mathrm{H} \alpha$ luminosity. These authors obtained (after adapting to a Kroupa IMF by multiplying by 1.59$)$ a relation

$S F R=10^{-9} L_{W 4}\left[M_{\odot} \mathrm{yr}^{-1}\right]$,

where $L_{W 4}=v f_{W 4} 4 \pi D^{2}$ is the luminosity in the $W 4$ band (in units of bolometric solar luminosity), where $f_{W 4}$ is the flux in this band. This relation is similar to that found by Jarrett et al. (2013). The values for the SFR are listed in Table 2.

\subsection{Stellar mass}

The near-IR wavelength range is dominated by emission from low-mass stars and is therefore a very good measure of the total stellar mass. We calculated the stellar mass from the luminosity in the WISE $W 1$ and $W 2$ bands (at $3.4 \mu \mathrm{m}$ and $4.6 \mu \mathrm{m}$ ), adopting Eq. (9) from Jarrett et al. (2013), i.e.,

$\log \left(\frac{M_{*}}{L_{W 1}}\right)=-0.246-2.100(W 1-W 2)$,

where $W 1$ and $W 2$ are given in magnitudes and $L_{W 1}$ is the total in-band luminosity, derived by multiplying the spectral luminosity $L_{W 1}=v f_{W 1} 4 \pi D^{2}$ by a factor of 22.883 . As explained in Jarrett et al. (2013), this factor accounts for the difference between the total solar luminosity and the in-band values as measured by WISE. We converted from the flux densities listed in Zucker et al. (2016) to magnitude using the relationship $m_{\mathrm{VEGA}}=-2.5 \log \left(\frac{f_{v}}{f_{v, 0}}\right)$ with $f_{v, 0}$ given by $306,682,170.663$, 29.0448 , and 8.2839 for bands $W 1, W 2, W 3$, and $W 4$, respectively, as outlined in Sect. IV.4 of the WISE All-sky Explanatory Supplement. The values of the stellar mass are listed in Table 2.

\section{Results}

The available data allow us to study the molecular gas fraction $\left(M_{\mathrm{mol}} / M_{*}\right)$ and the star formation efficiency $(S F E=$ $\left.S F R / M_{\text {mol }}\right)$ of the galaxies as a function of their activity class. For this, we distinguish galaxies based on their WISE classification from Zucker et al. (2016; mid-IR active, mid-IR quiescent, and mid-IR canyon galaxies) and search for differences between the three groups.

We also investigate the properties of galaxies lying in the IRTZ, defined by Alatalo et al. (2014b) as galaxies with WISE colors in the range $0.8<W 2(4.6 \mu \mathrm{m})-W 3(12 \mu \mathrm{m})<2.4$, corresponding to the transition region between active and quiescent galaxies. The data by Zucker et al. (2016) showed a systematic offset of 0.3-0.4 mag in W2 - W3 compared to the Alatalo sample (see Fig. 7 of Zucker et al. 2016). Zucker et al. argued that this offset is due to the different apertures used in the photometry applied to each sample. Alatalo et al. (2014b) used wgmag 
Table 2. Molecular gas mass, SFR, and stellar mass.

\begin{tabular}{lcccccc}
\hline \hline Galaxy & $\begin{array}{c}\text { Distance }^{a} \\
{[\mathrm{Mpc}]}\end{array}$ & $\begin{array}{c}\log \left(M_{\mathrm{mol}, 0}\right)^{b} \\
{\left[M_{\odot}\right]}\end{array}$ & $\begin{array}{c}\log \left(M_{\mathrm{mol}}\right)^{b} \\
{\left[M_{\odot}\right]}\end{array}$ & Ref. $^{d}$ & $\begin{array}{c}S_{F} R^{e} \\
{\left[M_{\odot} \mathrm{yr}^{-1}\right]}\end{array}$ & $\begin{array}{c}\log \left(M_{*}\right)^{f} \\
{\left[M_{\odot}\right]}\end{array}$ \\
\hline HCG01b & 146.6 & 9.23 & 9.35 & 1 & 0.63 & 10.61 \\
HCG02b & 62.1 & 8.92 & 9.19 & 4 & 6.08 & 9.65 \\
HCG04a & 114.9 & 10.09 & 10.49 & 4 & 23.45 & 10.49 \\
HCG05a & 174.0 & 9.99 & 10.24 & 1 & 1.60 & 10.99 \\
$\ldots$ & $\ldots$ & $\ldots$ & $\ldots$ & $\ldots$ & $\ldots$ & $\ldots$ \\
\hline
\end{tabular}

Notes. ${ }^{(b)}$ Distance derived from redshift with $H_{0}=70 \mathrm{~km} \mathrm{~s}^{-1} \mathrm{Mpc}^{-1}$. $^{(b)}$ Cold molecular gas within the central pointing, calculated from Eq. (2). ${ }^{(c)}$ Cold molecular gas mass, extrapolated from the central pointing to the entire disk, calculated as described in Sect. 2.2. ${ }^{(d)}$ References for the CO(1-0) measurements: (1) this work; (2) Lisenfeld et al. (2014); (3) Martinez-Badenes et al. (2012); (4) Leon et al. (1998); (5) Verdes-Montenegro et al. (1998); (6) Wiklind et al. (1995); and (7) Mirabel et al. (1990). ${ }^{(e)}$ Star formation rate, calculated from the WISE W4 band with Eq. (4). ${ }^{(f)}$ Stellar mass, calculated from the WISE $W 1$ and $W 2$ bands with Eq. (5). The full table is available at the CDS.

values from the ALLWISE source catalog, which is based on elliptical apertures scaled from the 2MASS extended source cata$\log$ apertures, whereas Zucker et al. used $1-3 \sigma$ contours derived from the averaged, $\lambda^{-1}$ weighted reference image of the first three WISE bands. The same offset was found by Cluver et al. (2014) between wgmag values and the use of isophotal contours. Taking this offset into account, the IRTZ defined by Alatalo et al. (2014b) shifts to $1.2<W 2(4.6 \mu \mathrm{m})-W 1(3.4 \mu \mathrm{m})<2.8$. We furthermore restrict the IRTZ to a more central zone in the $W 2-W 3$ distribution with a minimum in galaxy density between active and quiescent galaxies (see Fig. 7 in Zucker et al. 2016). Based on this figure, we define galaxies in the IRTZ as those with $1.4<W 2-W 3<2.6$.

\subsection{Molecular gas mass and stellar mass}

Figure 1 shows the molecular gas mass as a function of stellar mass for the galaxies in our sample, color-coded according to their class determined in Zucker et al. (2016). A broad correlation exists between both quantities. Active galaxies have stellar masses between about $\log \left(M_{*} / M_{\odot}\right) \sim 9-11$, whereas the mass range of quiescent galaxies is shifted to higher values $\left(\log \left(M_{*} / M_{\odot}\right) \sim 10-11.5\right)$. The masses of canyon galaxies are in an intermediate range $\left(\log \left(M_{*} / M_{\odot}\right) \sim 9.5-11.5\right)$. With respect to the molecular gas the distribution is remarkably stratified. Whereas for active galaxies the molecular gas mass is mostly between 10 and $100 \%$ of the stellar mass (with some objects having an even higher value), the molecular gas per stellar mass is considerably lower for quiescent galaxies (between 1 and 10\%). Canyon galaxies are right in between these two groups, suggesting that they present a transition between them.

Figure 2 shows the ratio between molecular and stellar mass as a function of morphological type. As expected, most active galaxies are late types and most quiescent galaxies are early types. Canyon galaxies are mostly late-type galaxies. We see again the distinct dichotomy in $M_{\mathrm{mol}} / M_{*}$ between active and quiescent galaxies, where canyon galaxies have values within the lower end of the active galaxies and span a similar range of morphological types.

In addition to distinguishing active, quiescent, and canyon galaxies, the lower panels also show galaxies belonging to the IRTZ. These have, as canyon galaxies, a lower value of $M_{\operatorname{mol}} / M_{*}$ than active galaxies, but are, to a larger extent, early-type galaxies and many are classified as quiescent galaxies by Zucker et al. (2016). About half of the canyon galaxies also belong to the IRTZ showing that both criteria probe a similar class of objects.

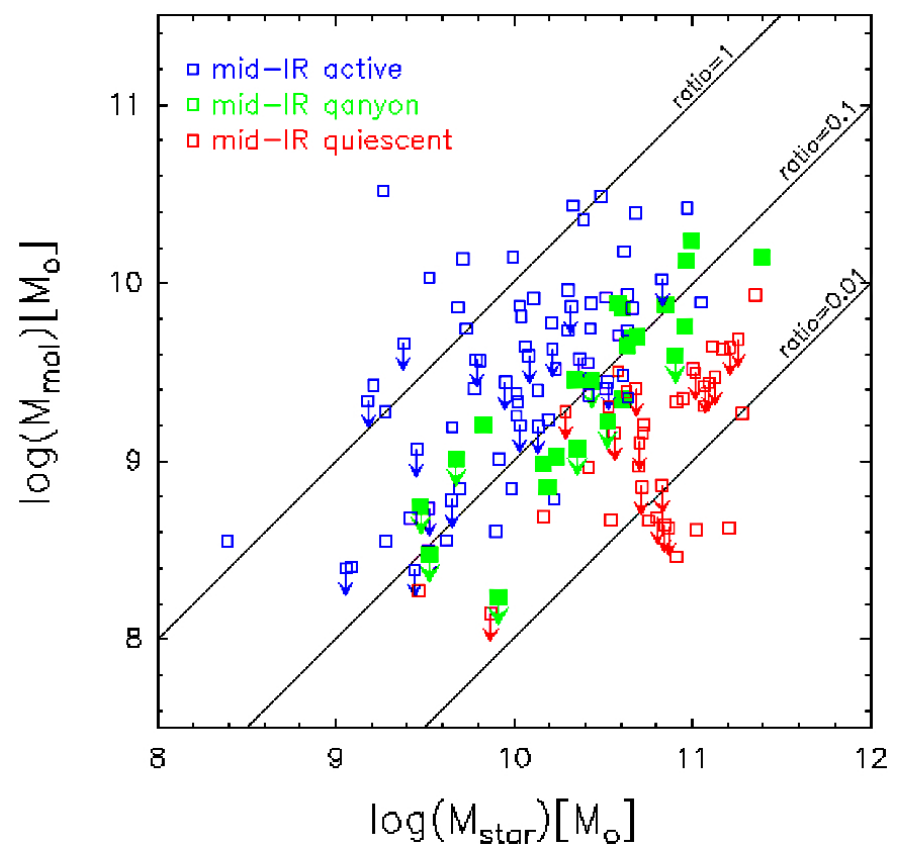

Fig. 1. Stellar mass, derived with Eq. (5), as a function of molecular gas mass. The lines show ratios of $M_{\mathrm{mol}} / M_{*}=100 \%, 10 \%$, and $1 \%$, respectively. The objects are color-coded according to the classification of Zucker et al. (2016) with the mid-IR active population in blue and the mid-IR quiescent population in red to follow the optical convention typically adopted in the literature.

The mean and median values for the various groups, calculated by taking into account the upper limits in the calculation ${ }^{5}$, are listed in Table 3. We list the values for the entire subsample and the values for only late-type galaxies to compare the means for the same morphological group. This is helpful to avoid mixing the effects of morphological type and activity class; earlytype galaxies are known to have a lower molecular gas content and SFR. The mean values confirm the visual impression from Fig. 2. Canyon and IRTZ galaxies have a significantly $(\sim 5-7 \sigma)$ lower $M_{\mathrm{mol}} / M_{*}$ than active galaxies. This result holds when only considering late-type galaxies $(\sim 5 \sigma)$.

5 When calculating the mean values we take upper limits into account with the program ASURV Rev (Astronomical Survival Analysis) Rev 1.1 (Lavalley et al. 1992), which is a generalized statistical package that implements the methods presented by Feigelson \& Nelson (1985). 


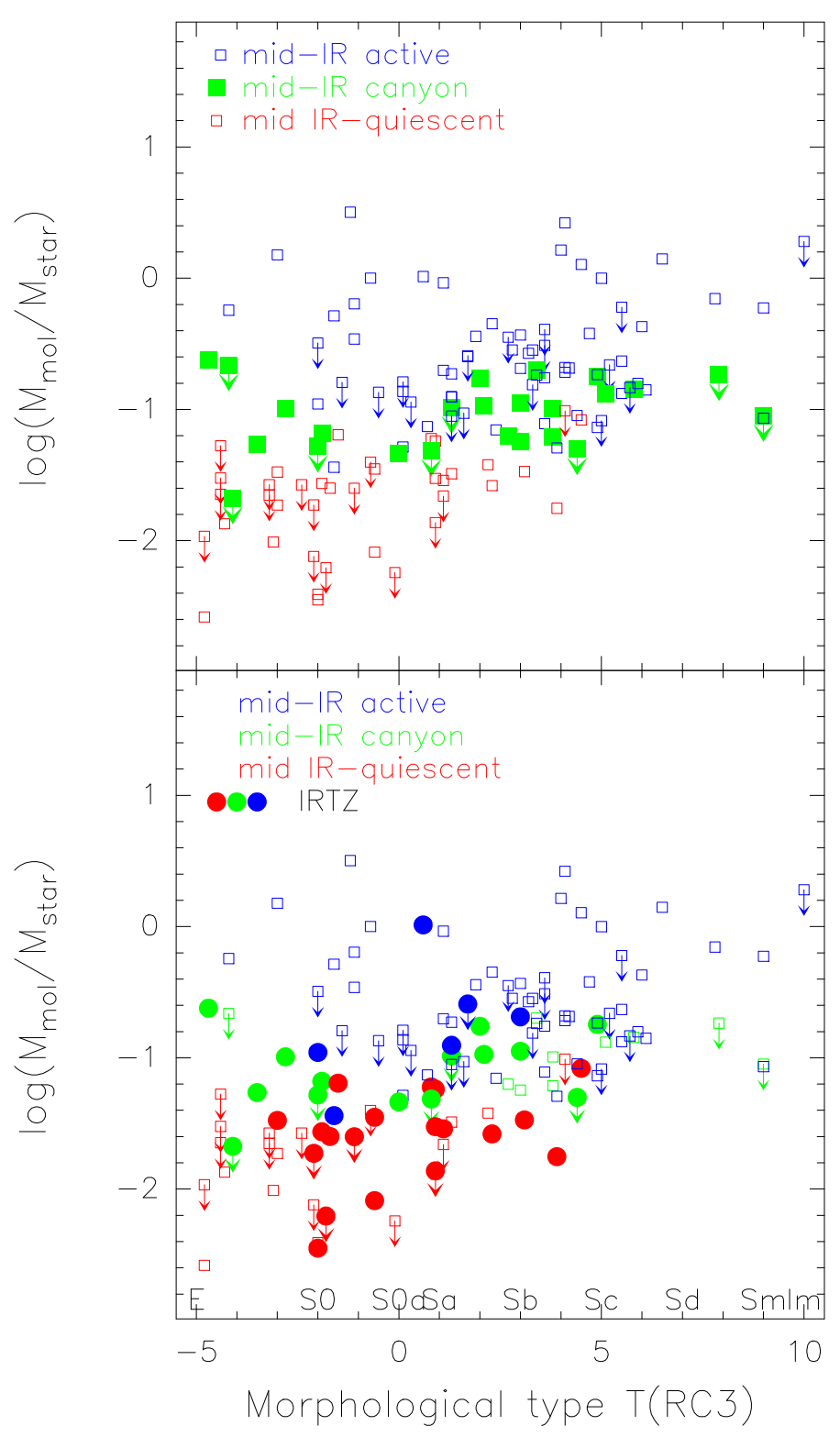

Fig. 2. Ratio of molecular gas mass to stellar mass as a function of morphological type. The color coding is as in Fig. 1. Filled circles (lower panel) denote galaxies belonging to the IRTZ.

\subsection{Molecular gas mass and star formation rate}

Figure 3 shows a comparison of the SFR as a function of molecular gas mass for active, quiescent, and canyon galaxies. We include the result (best-fit and standard deviation) of Bigiel et al. (2011) who found a linear relation between the SFR and molecular gas mass for a large sample of spiral and starburst galaxies. Active galaxies roughly follow this relation, albeit with a large scatter and a small offset $(\sim 0.1-0.2$ dex). Quiescent galaxies have a pronounced offset toward lower SFR for a given molecular gas mass and they lack the high molecular gas masses present in some active galaxies; the upper $\operatorname{limit}$ in $\log \left(M_{\text {mol }} / M_{\odot}\right)$ is $\approx 9.8$ for quiescent galaxies, whereas for active galaxies it is $\approx 10.5$. Canyon galaxies have a considerably lower SFR for a given molecular gas mass than active galaxies, in the same range as the quiescent galaxies.

Figure 4 shows the distribution of the SFE as a function of the morphological types. The mean and median values for the three groups are listed in Table 4.

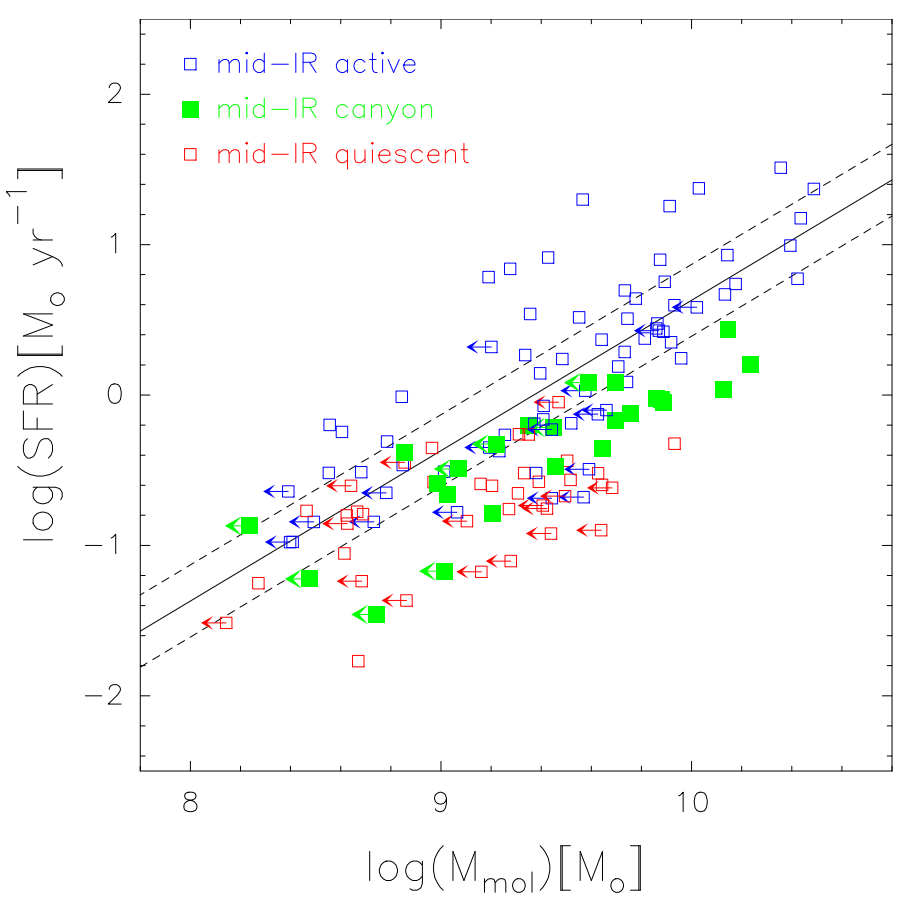

Fig. 3. Star formation rate vs. total molecular gas mass, both calculated as explained in Sect. 2. The color coding is as in Fig. 1. The lines show the mean value (full line) and dispersion (dashed lines) found by Bigiel et al. (2011) for a sample of spiral and starburst galaxies.

Table 3. Mean and median $\log \left(M_{\mathrm{mol}} / M_{*}\right)$ for different samples.

\begin{tabular}{lccc}
\hline \hline Class & Mean & Median & $n / n_{\text {up }}{ }^{a}$ \\
\hline Full sample & & & \\
Active & $-0.67 \pm 0.06$ & -0.74 & $68 / 17$ \\
Canyon & $-1.13 \pm 0.07$ & -1.20 & $24 / 8$ \\
IRTZ & $-1.44 \pm 0.10$ & -1.47 & $38 / 10$ \\
quiescent & $-1.91 \pm 0.09$ & -1.88 & $38 / 16$ \\
\hline Late-types galaxies $(T>0)$ & & & \\
Active & $-0.67 \pm 0.06$ & -0.73 & $52 / 12$ \\
Canyon & $-1.04 \pm 0.06$ & -1.10 & $16 / 5$ \\
IRTZ & $-1.21 \pm 0.11$ & -1.31 & $20 / 5$ \\
quiescent & $-1.50 \pm 0.07$ & -1.52 & $13 / 3$ \\
\hline
\end{tabular}

Notes. ${ }^{(a)}$ Total number of galaxies $(n)$ and number of upper limits $\left(n_{\mathrm{up}}\right)$.

The mean value for active galaxies lies slightly higher, but within the scatter of the mean value found by Bigiel et al. (2011) $(-9.37 \pm 0.23)$. Canyon and IRTZ galaxies have a clearly lower SFE than active galaxies $(>5 \sigma)$, both for the entire subsample and for late-type galaxies alone. Galaxies belonging to the IRTZ have very similar values for the SFE as the canyon galaxies. Galaxies classified as quiescent outside the IRTZ are mostly early types and have very low SFE and a low CO detection rate.

For late-type galaxies, all the canyon/IRTZ galaxies lie on the low end of the distribution of the SFE. Toward earlier types (Sa and earlier), the SFE of canyon/IRTZ becomes similar to the values of quiescent galaxies. This is in principle a surprising result, however, it should be taken with caution mainly because of the low SFRs of early-type galaxies. Any SFR indicator is less reliable for these low values (Leroy et al. 2012) and, in particular, the mid-IR dust emission because of the contribution of 
U. Lisenfeld et al.: The role of molecular gas in galaxy transition in compact groups

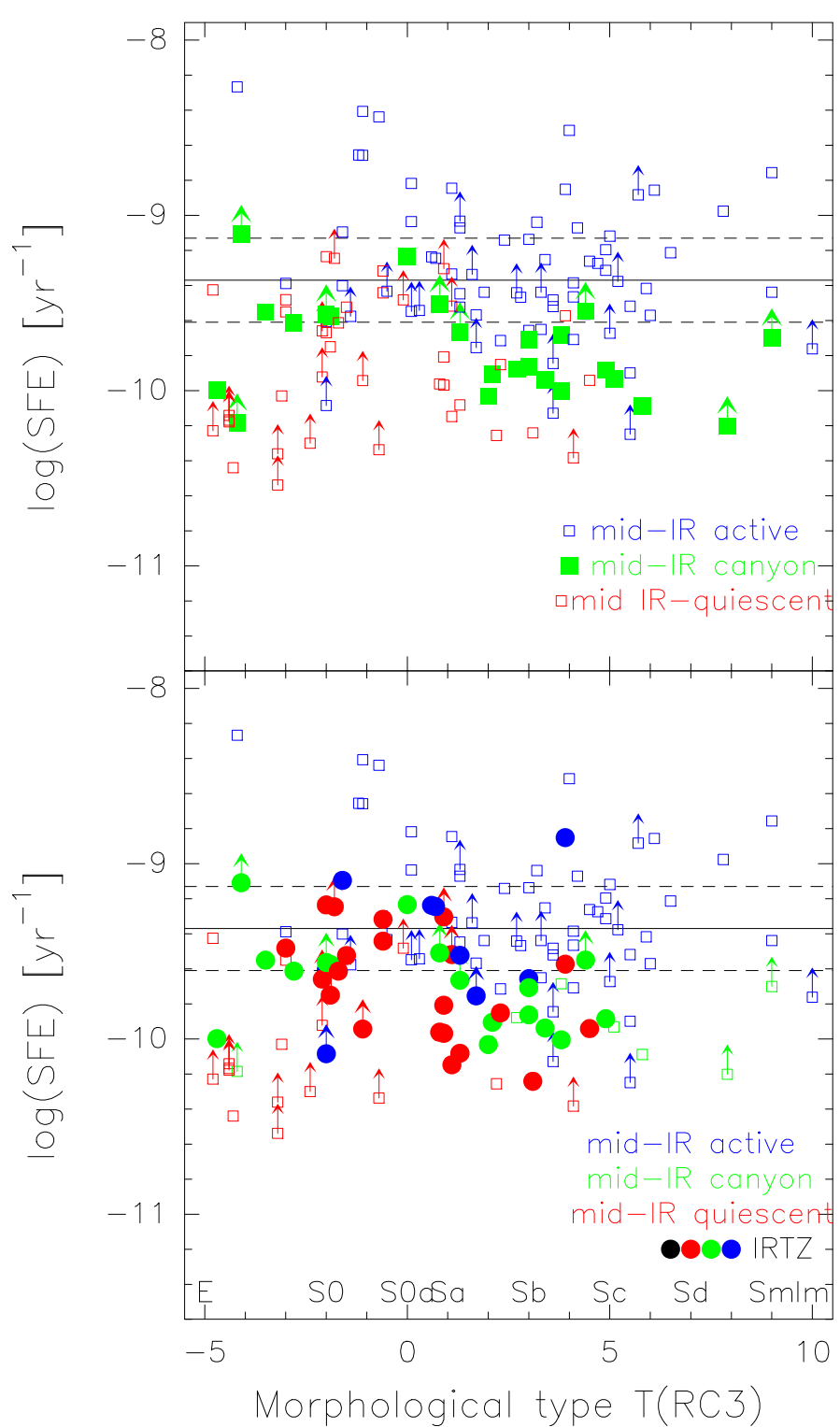

Fig. 4. SFE as a function of morphological type. The color coding is as in Fig. 1. Filled circles denote galaxies belonging to the IRTZ (lower panel). The lines show the mean value (full line) and dispersion (dashed lines) found by Bigiel et al. (2011) for a sample of spiral and starburst galaxies.

asymptotic giant branch stars to the mid-IR (Kaviraj et al. 2007). Lenkić et al. (2016) estimated the effect of this contamination for a sample of CG galaxies and found that for about $40 \%$ of quiescent galaxies the contribution of AGB stars to the $24 \mu \mathrm{m}$ emission could be $50 \%$ or more whereas for active the contribution of AGB stars was negligible. A comparision with a different SFR tracer would be desirable. We carry out this comparison in Sect. 4.1, but the number of canyon/IRTZ early-type galaxies available for this test is too low to draw any useful conclusions.

\subsection{Overall picture}

We have found that canyon/IRTZ galaxies have a molecular gas fraction $\left(M_{\mathrm{mol}} / M_{*}\right)$ in between active and quiescent galaxies and a SFE as low as quiescent galaxies. This suggests that canyon/IRTZ galaxies are indeed in transition between the active and quiescent phase. Their SFE is already as low as for quiescent
Table 4. Mean and median $\log (\mathrm{SFE})$ (in units of $\mathrm{yr}^{-1}$ ) for different samples.

\begin{tabular}{lccc}
\hline \hline Class & Mean & Median & $n / n_{\text {up }}{ }^{a}$ \\
\hline Full sample & & & \\
Active & $-9.17 \pm 0.05$ & -9.25 & $68 / 17$ \\
Canyon & $-9.65 \pm 0.07$ & -9.71 & $24 / 8$ \\
IRTZ & $-9.58 \pm 0.06$ & -9.59 & $38 / 10$ \\
quiescent & $-9.67 \pm 0.06$ & -9.61 & $38 / 16$ \\
\hline Late-type galaxies $(T>0)$ & & & \\
Active & $-9.26 \pm 0.05$ & -9.27 & $52 / 12$ \\
Canyon & $-9.80 \pm 0.05$ & -9.89 & $16 / 5$ \\
IRTZ & $-9.71 \pm 0.07$ & -9.85 & $20 / 5$ \\
quiescent & $-9.87 \pm 0.09$ & -9.96 & $13 / 3$ \\
\hline
\end{tabular}

Notes. ${ }^{(a)}$ Total number of galaxies $(n)$ and number of upper limits $\left(n_{\text {up }}\right)$.

galaxies, which might indicate similar physical properties of the molecular gas, but they still have a larger molecular gas content. We speculate that their future, in the absence of additional external influences, is to continue forming stars with a low efficiency and thereby continuously decreasing their molecular gas content.

\section{Discussion}

\subsection{Comparison of SFR and $M_{\star}$ to other studies}

We used a prescription to calculate the SFR and stellar mass entirely based on WISE data. In this way, we managed to maximize the sample size. As a test of the robustness of our results, we compared the values for the SFR and $M_{*}$ with those of Bitsakis et al. (2011) and Lenkić et al. (2016) and repeated our analysis with their values for those objects that are in common.

Bitsakis et al. (2011) carried out a multiwavelength analysis, based on data from the UV to near-infrared for a sample of 135 galaxies and additional mid- and far-IR from Spitzer and AKARI for a subsample. They used the model of da Cunha et al. (2008) to fit the SED and derive stellar mass, SFR, and extinction. We compared the values of $M_{*}$ and the SFR for those galaxies that were in common with our sample (112 objects in total, 84 objects detected in all WISE bands, and 66 galaxies detected by WISE and with CO data). Both the SFRs and stellar masses obtained by Bitsakis et al. (2011) correlate well and linearly with our values (correlation coefficients $r=0.67$ and 0.91 and bisector slopes $1.05 \pm 0.05$ and $0.99 \pm 0.13$, respectively). There is a small, constant offset between our SFR and $M_{*}$ and those derived by Bitsakis et al. (2011); our $M_{*}$ is about $50 \%$ lower than that of Bitsakis et al. (2011) and our SFR is about 30\% higher.

We reanalyzed for this smaller sample, consisting of objects in common with Bitsakis et al. (2011), using the SFR and $M_{*}$ derived by Bitsakis et al. (2011). The results confirmed the trends found with the larger, WISE-based sample with very similar values both for the SFE and molecular gas fraction. Thus, also for this smaller sample, the mean $\log \left(S F E / \mathrm{yr}^{-1}\right)$ for canyon galaxies $(-9.88 \pm 0.09$ for eight objects) and IRTZ galaxies $(-9.89 \pm 0.14$ for 14 objects) is significantly below the value for active galaxies $(-9.14 \pm 0.14$ for 40 objects), and the molecular gas fraction $\log \left(M_{\mathrm{mol}} / M_{*}\right)$ is lower in canyon $(-1.26 \pm 0.16)$ and IRTZ galaxies $(-1.79 \pm 0.12)$ than in active galaxies $(-0.93 \pm 0.7)$.

A different way to calculate the SFR in CG galaxies was employed by Lenkić et al. (2016). They derived the SFR for a 
sample of 175 galaxies based on Spitzer MIPS data and UV data from Swift UVOT. Their derivation of the SFR is thus sensitive to both the dust-enshrouded and unextincted SF. There exists a good correlation between the Lenkić et al. (2016) SFR and our SFR (correlation coefficient $r=0.87$ ) for the 53 galaxies that are in common in both samples, have both UV and Spitzer $24 \mu \mathrm{m}$ data and are detected in all WISE bands. The slope is, however, not unity, and at low SFRs the values of Lenkić et al. (2016) are about a factor of $\sim 3$ higher than those of Zucker et al. (2016), indicating that the UV emission is, especially at low SFRs, important to trace the total SFR.

We reanalyzed the subsample of 42 galaxies that are in common and have CO data using the (UV+24 $\mu \mathrm{m})$ SFR from Lenkić et al. (2016). The results were inconclusive. At high molecular gas masses (above $\sim 10^{9} M_{\odot}$ ) the SFE of canyon/IRTZ ( 3 objects in each group) is $0.5-1.0$ dex below the value of active galaxies, consistent with our results. However, at lower masses, the 3 canyon and 4 IRTZ have SFEs in the range of active galaxies. A larger sample of HCG galaxies with UV data, especially at low masses, is needed in order to perform a statistically significant test.

\subsection{Role of molecular gas in galaxy transition}

Our analysis has shown that the molecular gas is a crucial factor in the transition of galaxies from actively star forming to quiescent. Both the molecular gas fraction, $M_{\mathrm{mol}} / M_{*}$, and the SFE decrease significantly in the transition phase (canyon/IRTZ galaxies). This means that in the IR transition phase the molecular gas content becomes lower and the remaining molecular gas forms stars less efficiently. In the following we discuss possible causes for these findings.

A loss of atomic gas is a well-known process in CGs with deficiencies reaching very high values (more than 90\%) in some cases (Verdes-Montenegro et al. 2001). However, the molecular gas does not seem to be strongly affected by this. Previous surveys of the molecular gas (Leon et al. 1998; Verdes-Montenegro et al. 1998) found no strong decrease in the molecular gas compared to field galaxies. Martinez-Badenes et al. (2012) compared molecular and atomic gas deficiencies in a sample of HCG late-type galaxies and found no significant correlation; even galaxies that were strongly deficient in HI can show a normal $\mathrm{H}_{2}$ content. These authors explained this different behavior with the more extended distribution of HI compared to $\mathrm{H}_{2}$. This means that overall there is no lack of molecular gas in late-type galaxies in HCG and that this deficiency is only present for IR transitioning galaxies.

The lack of molecular gas could either be due to a decrease in the formation of $\mathrm{H}_{2}$, the destruction of the molecular gas, or a loss of the molecular gas, for example, by tidal forces, in a similar way as HI. So far it is unclear what the relevant process is. We can only exclude a lack of atomic gas supply as a reason because observationally there is no relation between a deficiency in $\mathrm{HI}$ and $\mathrm{H}_{2}$. The formation rate of the $\mathrm{H}_{2}$ is proportional to the gas density, which might be lower in the transition phase. A low gas density would at the same time explain the low SFE (see below). In the transition phase tidal forces might also be strong enough to affect the more central areas of the galaxies where molecular gas is present. If tidal forces were the relevant process, we would expect to observe molecular gas outside the galaxies or in tidal arms or streams. Thus, further observations of the molecular gas distribution and kinematics are necessary to distinguish between the different processes.
The low SFE of canyon and IRTZ galaxies shows that a lack of molecular gas is not the only reason for a decrease in SF during the transition. Instead, the remaining molecular gas has lost its ability to form stars. A possible reason could be a perturbation of the molecular gas, possibly due to turbulence injected by shocks that are produced by the interaction with neighboring galaxies or intra-group gas. This has been seen in the case of HCG 57a, which is a galaxy in close interaction with HCG 56d, where interferometric CO data has shown the presence of perturbed molecular gas that might explain its low SFE (Alatalo et al. 2014a). Collision with the IGM has been suggested by Cluver et al. (2013) to cause enhanced warm $\mathrm{H}_{2}$ emission. The best-studied example for this kind of process is HCG 92 (Stephan's Quintet), where shocks and turbulence are strongly suggested to be responsible for the suppression of SF in the intra-group gas (Cluver et al. 2010; Appleton et al. 2013, 2017). Furthermore, Bitsakis et al. (2016) found indications for the presence of shocks in CG galaxies below the SF main sequence.

Our results allow us to provide additional pieces of evidence to understand the evolution of galaxies in CGs. Altogether, the following evolutionary sequence seems to be likely (see also Verdes-Montenegro et al. 2001; Walker et al. 2010, 2012; Cluver et al. 2013; Alatalo et al. 2014b; Bitsakis et al. 2016, who suggested similar models). In an early stage, the atomic gas of galaxies in CGs gets extracted from the galaxies from tidal forces and gets distributed in the intra-group medium (Verdes-Montenegro et al. 2001; Borthakur et al. 2010). The outer parts of galaxies are affected first, such that the molecular gas and dust (Bitsakis et al. 2014) are not (much) depleted in this phase. This explains the lack of molecular gas deficiency in $\mathrm{CG}$ galaxies in general. As CGs evolve, the velocity dispersion increases and interactions, such as tidal interactions between galaxies and interactions with the intra-group gas, become more important. At this stage, the molecular gas content and SFE become affected.

Although in this scenario the decrease of the molecular gas is not causally related to a lack of atomic gas, there is a temporal sequence and we would expect transitioning galaxies with a low molecular gas content to be deficient in $\mathrm{HI}$ as well. The necessary high-resolution HI data are available for 21 transitioning galaxies ( 3 canyon and 18 IRTZ galaxies; see Table 5 in Martinez-Badenes et al. 2012) and confirm our expectation. Fourteen galaxies have $10 \%$ or less of the expected HI mass, six galaxies have between 10 and $30 \%$, and only one galaxies has a slight deficiency $(60 \%$ of the expected HI).

\subsection{Relevance for galaxy evolution}

The cosmic evolution of galaxies has been characterized by a strong decrease in the SFR since about $z \sim 1-1.5$ (Madau et al. 1998; Hopkins \& Beacom 2006); the reason for this is still an open question. There has been a large effort in recent years to better understand the cosmic SF history with different approaches. One way is to study the so-called galaxy main sequence (MS), which is the relation between the SFR and stellar mass followed by star-forming galaxies (Noeske et al. 2007). The slope of this relation gives information about how the specific $S F R, s S F R=S F R / M_{*}$, changes as a function of stellar mass. There are indications that the slope decreases at high mass, i.e., that the SSFR is lower for massive galaxies, thus implying a quenching of SF at high masses. The turnover mass increases from $\sim 10^{10} M_{\odot}$ at $z \sim 0$ (Brinchmann et al. 2004) to $\sim 5 \times 10^{10}$ at $z \sim 1$ (Schreiber et al. 2016). 
In order to understand the possible causes of this quenching and cosmic SF history in general, measurements of the gas mass are necessary and it is useful to quantify the SFE and the (molecular or total) gas fraction as a function of redshift. Since it is observationally difficult to measure the gas content for distant galaxies, not many studies have been performed thus far. Combes et al. (2013) observed a sample of 39 ultraluminous IR galaxies (ULIRGs) with $z \sim 0-1$ in $\mathrm{CO}(1-0)$ and found a decrease of both the SFE and the molecular gas fraction for decreasing $z$. These authors concluded that both factors seems to be relevant for the decrease of the cosmic SF history. Schreiber et al. (2016) derived the total gas mass from Herschel dust data for a sample of galaxies in the CANDELS field (at $z \sim 1$ ) and found that for $M_{*} \gtrsim 5 \times 10^{10}$ both the sSFR and the SFE decreases. The total gas fraction showed no similar decline, indicating that a lack of gas is not the reason for the low SSFR. These authors suggested that the decrease in SFE implies a slow quenching (the cause of which is unclear) such that galaxies decrease their SFR slowly while remaining on the MS, instead of suffering a catastrophic event like a merger. A decrease in the SFE with stellar mass was also found by Saintonge et al. (2011b) for local, mass-selected galaxies and a weak decrease in the molecular gas fraction was found by Saintonge et al. (2011a).

When we compare these results to ours, we need to keep in mind that the CG galaxies live in a special environment where interactions, both between galaxies and with the intragroup medium, play a crucial role. In spite of the interaction, the galaxies in our sample are, however, not in a starburst mode of SF; instead they form stars in a normal way, similar to spiral galaxies. Because of this, the mean SFE of the active group is similar to the value of Bigiel et al. (2011) for spiral galaxies. Extreme starbursts have much higher SFEs (e.g., Daddi et al. 2010). Similarly, the majority of actively star-forming CG galaxies in the study of Lenkic et al. (2016) was consistent with the local MS in the parameter space of SFR versus $M_{*}$ found by Chang et al. (2015). Furthermore, their molecular gas surface density $\left(\lesssim 50 M_{\odot} \mathrm{pc}^{2}\right)$, is much lower than the surface density of $\gtrsim 100 M_{\odot} \mathrm{pc}^{2}$ typical for starbursts.

All these studies identified a decrease of the SFE as a reason for the quenching of SF. It is interesting that this result holds for very different sample, from ULIRGs which form stars in a starburst mode, to normal galaxies. For our sample we speculate that the environment is responsible for the decrease in SFE. It is still unclear, but important to find out, whether the decrease of SFE in the other samples has the same origin. The finding by Schreiber et al. (2016) that the decrease in the gas fraction is not related to the quenching of SF contrasts with our results and is most likely due to the difference in environment, in particular the dense, but low-mass environment of our sample.

\section{Conclusions and summary}

We analyzed the molecular gas content based on $\mathrm{CO}(1-0)$ data from the literature and from our own new data IRAM $30 \mathrm{~m}$ observations for a sample of 130 galaxies in CGs with detections from the WISE satellite in all four bands. The midIR WISE colors were used by Zucker et al. (2016) to classify these galaxies into actively star-forming, quiescent, and canyon (i.e., transitioning between both phases) galaxies. We used these data and classifications to compare the SFE and molecular gas fraction, $M_{\mathrm{mol}} / M_{*}$, between the active, quiescent, and transitioning galaxies. We considered two types of transitioning galaxies: Canyon (Zucker et al. 2016) and IRTZ galaxies
(Alatalo et al. 2014b), selected based on the WISE [W2 - W3] color.

We found a significantly lower molecular gas fraction for canyon and IRTZ galaxies than in active galaxies, but still higher than in quiescent galaxies. This indicates that the transition from active to quiescent goes along with a decrease in molecular gas and that canyon/IRTZ galaxies are indeed in a transition process. The reason for this decrease is not entirely clear. A lack of gas supply can be excluded because the deficiencies in $\mathrm{HI}$ and $\mathrm{H}_{2}$ do not correlate (Martinez-Badenes et al. 2012). Possible causes are a decrease in the gas density (e.g., due to a perturbation of the gas), making molecular gas formation less efficient, or the tidal removal of molecular gas.

We found a significantly lower SFE for canyon and IRTZ galaxies than for active galaxies, which is comparable to the mean value of quiescent galaxies. This shows that the remaining molecular gas has lost its ability to form stars efficiently in the transition. A perturbation of the molecular gas, possibly from shocks produced by the interaction with neighboring galaxies or intra-group gas is a likely reason.

Thus, we found that molecular gas data can provide a crucial clue to the conditions of environmentally driven galaxy evolution in CGs. Based on these new data, we propose a possible scenario in line and extending conclusions from previous studies (e.g., Verdes-Montenegro et al. 2001; Walker et al. 2010, 2012; Cluver et al. 2013; Alatalo et al. 2014b; Bitsakis et al. 2016). for the evolution of galaxies in HCGs. In a first phase, galaxies lose their atomic gas, sometimes to a dramatic extent. Eventually, when the galaxies are in the canyon/IRTZ phase the molecular content and SFE also decrease. In this phase, the ISM is strongly affected, and this is most likely due to interaction with neighboring galaxies and/or the intra-group environment.

Acknowledgements. We appreciate very much the useful thoughts and comments from the referee. U.L. acknowledges support by the research projects AYA201453506-P from the Spanish Ministerio de Economía y Competitividad, from the European Regional Development Funds (FEDER) and the Junta de Andalucía (Spain) grants FQM108. We acknowledge the usage of the HyperLeda database (http://leda.univ-lyon1.fr) and of the Nasa Extragalactic Database (NED, https://ned.ipac.caltech.edu). This work is based on observations carried out under project number 167-16 with the IRAM $30 \mathrm{~m}$ telescope. IRAM is supported by INSU/CNRS (France), MPG (Germany), and IGN (Spain). We would like to thank the IRAM staff at the $30 \mathrm{~m}$ telescope warmly for their support during the observations.

\section{References}

Alatalo, K., Appleton, P. N., Lisenfeld, U., et al. 2014a, ApJ, 795, 159 Alatalo, K., Cales, S. L., Appleton, P. N., et al. 2014b, ApJ, 794, L13 Alatalo, K., Appleton, P. N., Lisenfeld, U., et al. 2015, ApJ, 812, 117 Appleton, P. N., Guillard, P., Boulanger, F., et al. 2013, ApJ, 777, 66 Appleton, P. N., Guillard, P., Togi, A., et al. 2017, ApJ, 836, 76

Baldry, I. K., Glazebrook, K., Brinkmann, J., et al. 2004, ApJ, 600, 681 Barton, E., Geller, M., Ramella, M., Marzke, R. O., \& da Costa, L. N. 1996, AJ, 112,871

Bell, E. F., Wolf, C., Meisenheimer, K., et al. 2004, ApJ, 608, 752 Bigiel, F., Leroy, A. K., Walter, F., et al. 2011, ApJ, 730, L13 Bitsakis, T., Charmandaris, V., da Cunha, E., et al. 2011, A\&A, 533, A142 Bitsakis, T., Charmandaris, V., Appleton, P. N., et al. 2014, A\&A, 565, A25 Bitsakis, T., Dultzin, D., Ciesla, L., et al. 2016, MNRAS, 459, 957 Borthakur, S., Yun, M. S., \& Verdes-Montenegro, L. 2010, ApJ, 710, 385 Brinchmann, J., Charlot, S., White, S. D. M., et al. 2004, MNRAS, 351, 1151 Chang, Y.-Y., van der Wel, A., da Cunha, E., \& Rix, H.-W. 2015, ApJS, 219, 8 Cluver, M. E., Jarrett, T. H., Kraan-Korteweg, R. C., et al. 2010, ApJ, 725, 1550 Cluver, M. E., Appleton, P. N., Ogle, P., et al. 2013, ApJ, 765, 93 Cluver, M. E., Jarrett, T. H., Hopkins, A. M., et al. 2014, ApJ, 782, 90 Combes, F., García-Burillo, S., Braine, J., et al. 2013, A\&A, 550, A41 da Cunha, E., Charlot, S., \& Elbaz, D. 2008, MNRAS, 388, 1595 Daddi, E., Elbaz, D., Walter, F., et al. 2010, ApJ, 714, L118 
Davis, T. A., Alatalo, K., Bureau, M., et al. 2013, MNRAS, 429, 534

Faber, S. M., Willmer, C. N. A., Wolf, C., et al. 2007, ApJ, 665, 265

Feigelson, E. D., \& Nelson, P. I. 1985, ApJ, 293, 192

Hickson, P. 1982, ApJ, 255, 382

Hickson, P., Mendes de Oliveira, C., Huchra, J. P., \& Palumbo, G. G. 1992, ApJ, 399,353

Hopkins, A. M., \& Beacom, J. F. 2006, ApJ, 651, 142

Jarrett, T. H., Masci, F., Tsai, C. W., et al. 2013, AJ, 145, 6

Johnson, K. E., Hibbard, J. E., Gallagher, S. C., et al. 2007, AJ, 134, 1522

Kaviraj, S., Kirkby, L. A., Silk, J., \& Sarzi, M. 2007, MNRAS, 382, 960

Lavalley, M. P., Isobe, T., \& Feigelson, E. D. 1992, in BAAS, 24, 839

Lee, J. C., Hwang, H. S., \& Ko, J. 2013, ApJ, 774, 62

Lenkić, L., Tzanavaris, P., Gallagher, S. C., et al. 2016, MNRAS, 459, 2948

Leon, S., Combes, F., \& Menon, T. K. 1998, A\&A, 330, 37

Leroy, A. K., Walter, F., Brinks, E., et al. 2008, AJ, 136, 2782

Leroy, A. K., Bigiel, F., de Blok, W. J. G., et al. 2012, AJ, 144, 3

Lisenfeld, U., Espada, D., Verdes-Montenegro, L., et al. 2011, A\&A, 534, A102

Lisenfeld, U., Appleton, P. N., Cluver, M. E., et al. 2014, A\&A, 570, A24

Madau, P., Pozzetti, L., \& Dickinson, M. 1998, ApJ, 498, 106

Makarov, D., Prugniel, P., Terekhova, N., Courtois, H., \& Vauglin, I. 2014, A\&A, 570, A13
Martinez-Badenes, V., Lisenfeld, U., Espada, D., et al. 2012, A\&A, 540, A96 Mirabel, I. F., Booth, R. S., Johansson, L. E. B., Garay, G., \& Sanders, D. B. 1990, A\&A, 236, 327

Nishiyama, K., Nakai, N., \& Kuno, N. 2001, PASJ, 53, 757

Noeske, K. G., Weiner, B. J., Faber, S. M., et al. 2007, ApJ, 660, L43

Ogle, P., Boulanger, F., Guillard, P., et al. 2010, ApJ, 724, 1193

Regan, M. W., Thornley, M. D., Helfer, T. T., et al. 2001, ApJ, 561, 218

Saintonge, A., Kauffmann, G., Kramer, C., et al. 2011a, MNRAS, 415, 32

Saintonge, A., Kauffmann, G., Wang, J., et al. 2011b, MNRAS, 415, 61

Schawinski, K., Urry, C. M., Simmons, B. D., et al. 2014, MNRAS, 440, 889

Schreiber, C., Elbaz, D., Pannella, M., et al. 2016, A\&A, 589, A35

Tzanavaris, P., Hornschemeier, A. E., Gallagher, S. C., et al. 2010, ApJ, 716, 556

Verdes-Montenegro, L., Yun, M. S., Perea, J., del Olmo, A., \& Ho, P. T. P. 1998, ApJ, 497, 89

Verdes-Montenegro, L., Yun, M. S., Williams, B. A., et al. 2001, A\&A, 377, 812

Walker, L. M., Johnson, K. E., Gallagher, S. C., et al. 2010, AJ, 140, 1254

Walker, L. M., Johnson, K. E., Gallagher, S. C., et al. 2012, AJ, 143, 69

Walker, L. M., Butterfield, N., Johnson, K., et al. 2013, ApJ, 775, 129

Wiklind, T., Combes, F., \& Henkel, C. 1995, A\&A, 297, 643

Zucker, C., Walker, L. M., Johnson, K., et al. 2016, ApJ, 821, 113 
Appendix A: Figures of spectra
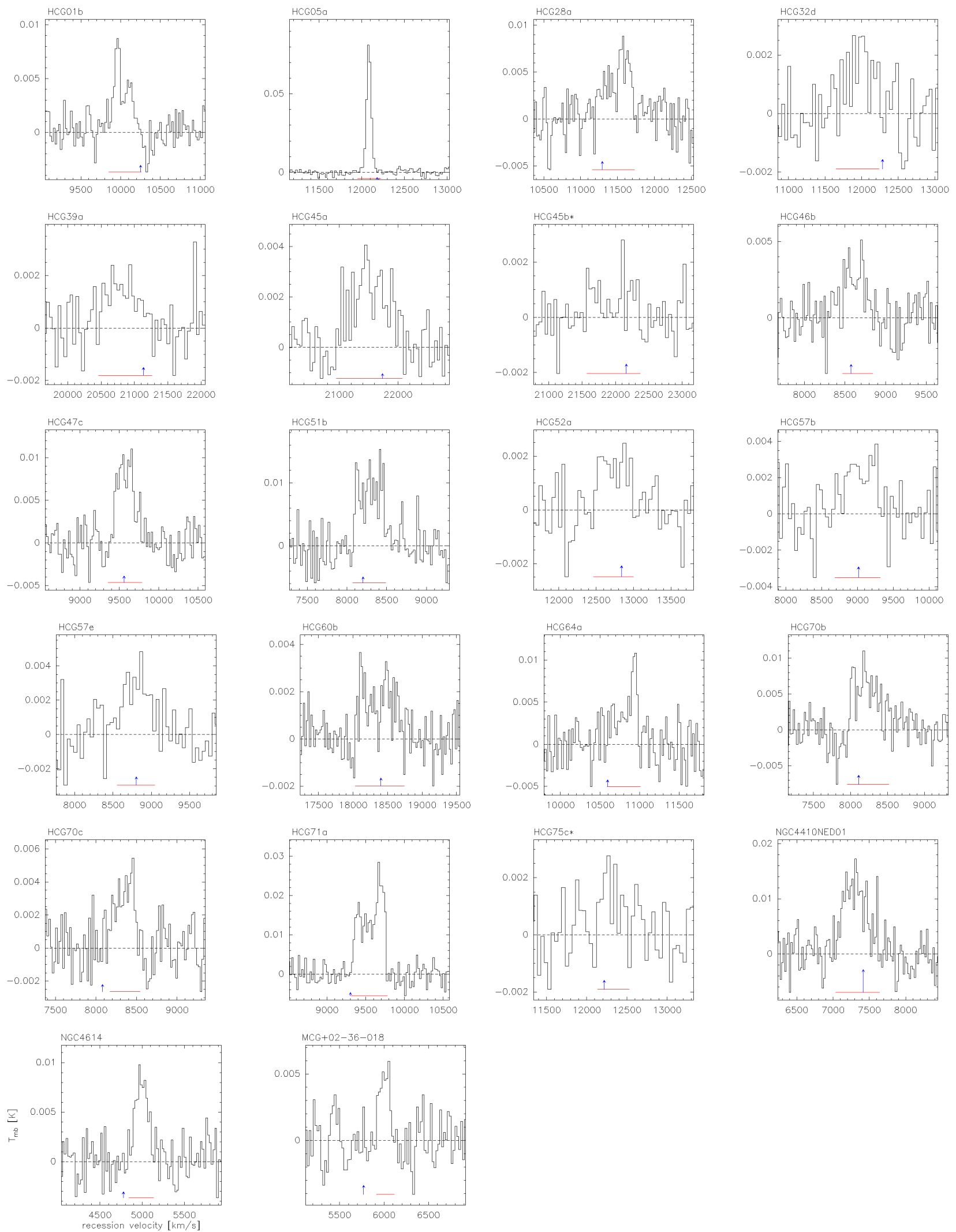

Fig. A.1. $\mathrm{CO}(1-0)$ spectra of the detected spectra (including tentative detections). The velocity resolution is $\sim 20 \mathrm{~km} \mathrm{~s}^{-1}$ for most spectra and $\sim 40 \mathrm{~km} \mathrm{~s}^{-1}$ for some cases where a lower resolution was required to clearly see the line. The red line segment shows the zero-level linewidth of the $\mathrm{CO}$ line adopted for the determination of the velocity integrated intensity. The blue upright arrow indicated the optical heliocentric recession velocity. An asterisk next to the name indicates a tentative detection. 


\section{Appendix B: Results of a WISE-selected subsample}

As outlined in Sect. 2, we carried out the entire analysis for a WISE selected subsample to check whether the CO-driven selection has produced a bias. We selected this subsample by including only those groups in which all galaxies with reliable WISE data (here $S / N>2.5 \sigma$ ) also had CO data. This subsample consists of 89 galaxies out of the full 130 galaxy sample used for analysis in the main body of the text.

In Figs. B.1 and B.2 we show the SFE and molecular gas content as a function of morphological type for this subsample, analogous to Figs. 2 and 4 for the full sample. The trend seen for the full sample is also present for the WISE-selected subsample. In Tables B. 1 and B. 2 we list the results for the mean and median values for the different subgroups, which entirely confirm the results for the full sample.

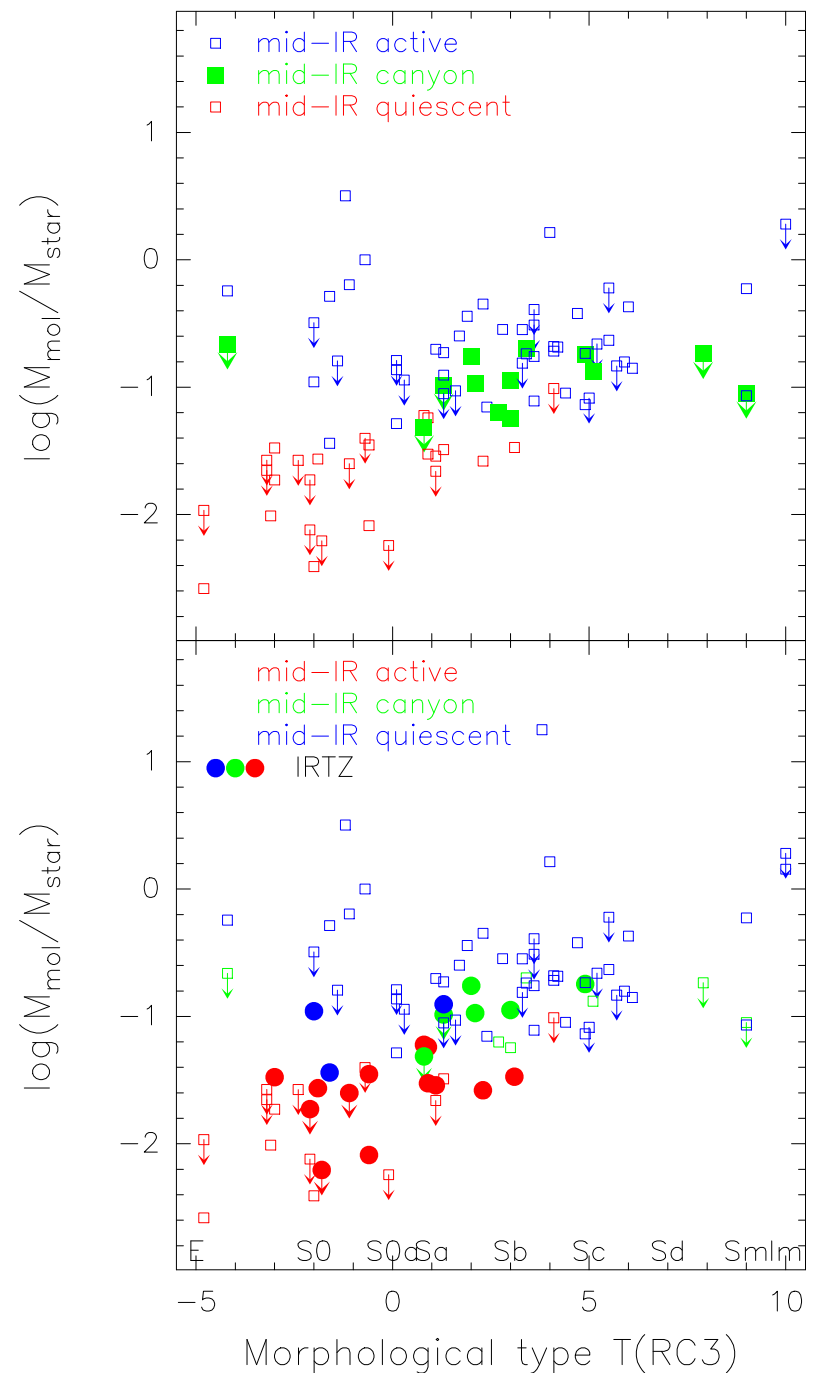

Fig. B.1. Ratio of molecular gas mass to stellar mass as a function of morphological type for the WISE-selected subsample of 89 galaxies with both high-quality WISE and CO data. The color coding is as in Fig. 1. Filled circles (lower panel) denote galaxies belonging to the IRTZ.

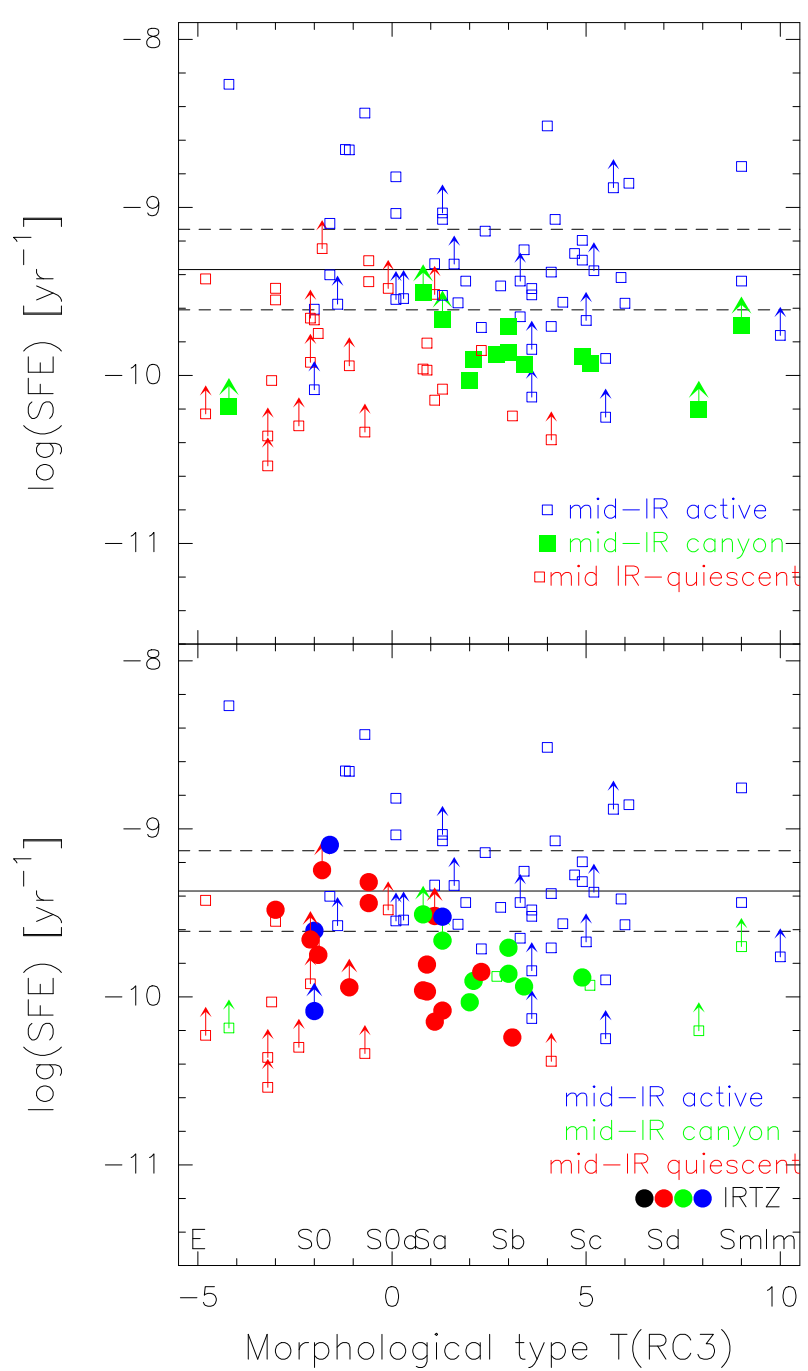

Fig. B.2. SFE as a function of morphological type for the WISEselected subsample of 89 galaxies with both high-quality WISE and $\mathrm{CO}$ data. The color coding is as in Fig. 1. Filled circles denote galaxies belonging to the IRTZ (lower panel). The lines show the mean value (full line) and dispersion (dashed lines) found by Bigiel et al. (2011) for a sample of spiral and starburst galaxies.

Table B.1. Mean and median $\log \left(M_{\mathrm{mol}} / M_{*}\right)$ for different groups of the WISE-selected subsample

\begin{tabular}{lccc}
\hline \hline Class & \multicolumn{1}{c}{ Mean } & Median & $n / n_{\mathrm{up}}{ }^{a}$ \\
\hline \multicolumn{2}{l}{ WISE-selected subsample, all morphological types } & \\
Active & $-0.76 \pm 0.06$ & -0.77 & $49 / 14$ \\
Canyon & $-1.03 \pm 0.07$ & -1.10 & $13 / 5$ \\
IRTZ & $-1.44 \pm 0.10$ & -1.48 & $22 / 5$ \\
quiescent & $-1.96 \pm 0.10$ & -2.05 & $27 / 12$ \\
\hline WISE-selected subsample, late types $(T>0)$ & \\
Active & $-0.76 \pm 0.06$ & -0.75 & $36 / 10$ \\
Canyon & $-1.03 \pm 0.07$ & -1.10 & $12 / 4$ \\
IRTZ & $-1.22 \pm 0.09$ & -1.31 & $13 / 2$ \\
quiescent & $-1.47 \pm 0.05$ & -1.52 & $9 / 2$ \\
\hline
\end{tabular}

Notes. ${ }^{(a)}$ Total number of galaxies $(n)$ and number of upper limits $\left(n_{\mathrm{up}}\right)$. 
Table B.2. Mean and median $\log (\mathrm{SFE})$ (in units of $\mathrm{yr}^{-1}$ ) for different groups of the WISE-selected subsample.

\begin{tabular}{lccc}
\hline \hline Class & \multicolumn{1}{c}{ Mean } & Median & $n / n_{\mathrm{up}}{ }^{a}$ \\
\hline \multicolumn{4}{l}{ WISE-selected subsample, all morphological types } \\
Active & $-9.17 \pm 0.07$ & -9.28 & \\
Canyon & $-9.79 \pm 0.06$ & -9.89 & $49 / 4$ \\
IRTZ & $-9.66 \pm 0.08$ & -9.77 & $13 / 5$ \\
quiescent & $-9.67 \pm 0.07$ & -9.68 & $22 / 5$ \\
\hline WISE-selected subsample, late types $(T>0)$ & \\
Active & $-9.28 \pm 0.07$ & -9.36 & $36 / 10$ \\
Canyon & $-9.79 \pm 0.06$ & -9.89 & $12 / 4$ \\
IRTZ & $-9.86 \pm 0.06$ & -9.90 & $13 / 2$ \\
quiescent & $-9.95 \pm 0.08 \mathrm{k}$ & -9.97 & $9 / 2$ \\
\hline
\end{tabular}

Notes. ${ }^{(a)}$ Total number of galaxies $(n)$ and number of upper limits $\left(n_{\mathrm{up}}\right)$.

Table C.1. Mean and median value of the aperture correction, $f_{\text {aper }}$.

\begin{tabular}{lccc}
\hline \hline Class & Mean & Median & $n^{a}$ \\
\hline Total & $1.70 \pm 0.06$ & 1.51 & 130 \\
Active & $1.64 \pm 0.08$ & 1.40 & 68 \\
Canyon & $1.65 \pm 0.10$ & 1.59 & 24 \\
IRTZ & $1.73 \pm 0.12$ & 1.58 & 38 \\
Quiescent & $1.85 \pm 0.13$ & 1.60 & 38 \\
\hline
\end{tabular}

Notes. ${ }^{(a)}$ Total number of galaxies $(n)$.

\section{Appendix C: Effect of the aperture correction}

We applied an aperture correction, $f_{\text {aper }}$, to our $\mathrm{CO}$ measurements that is based on the ratio between optical diameter and beam size. In order to test whether this aperture correction could have produced a bias in the results, we carried out two tests.

We compared the mean and median value of $f_{\text {aper }}$ for the different galaxy groups to search for possible systematic differences that might cause a bias. Table C.1 lists the results and shows that the mean and median values are comparable for the four groups. The histogram of the distributions, shown in Fig. C.1, confirms this result and shows that all groups have a similar distribution that is strongly skewed toward values of $f_{\text {aper }}<2$. There are differences, most noticeably between active and canyon galaxies, with the former having a maximum for galaxies $1.25<f_{\text {aper }}<1.5$, whereas canyon galaxies have more galaxies in the bin $1.5<f_{\text {aper }}<1.75$. This difference is however too small to produces a serious bias in our results.

As a second test, we carried out our analysis for a subsample with a small aperture correction, $f_{\text {aper }}<1.6$, but with a large enough number of galaxies to obtain statistically significant results. The distribution of $M_{\mathrm{mol}} / M_{*}$ and the SFE as a function of morphological type are shown in Figs. C.2 and C.3, and the results for the corresponding mean and median values are shown in Tables C. 2 and C.3. The results are entirely consistent with those of the total sample, showing that the application of the aperture correction has not introduced any biases.

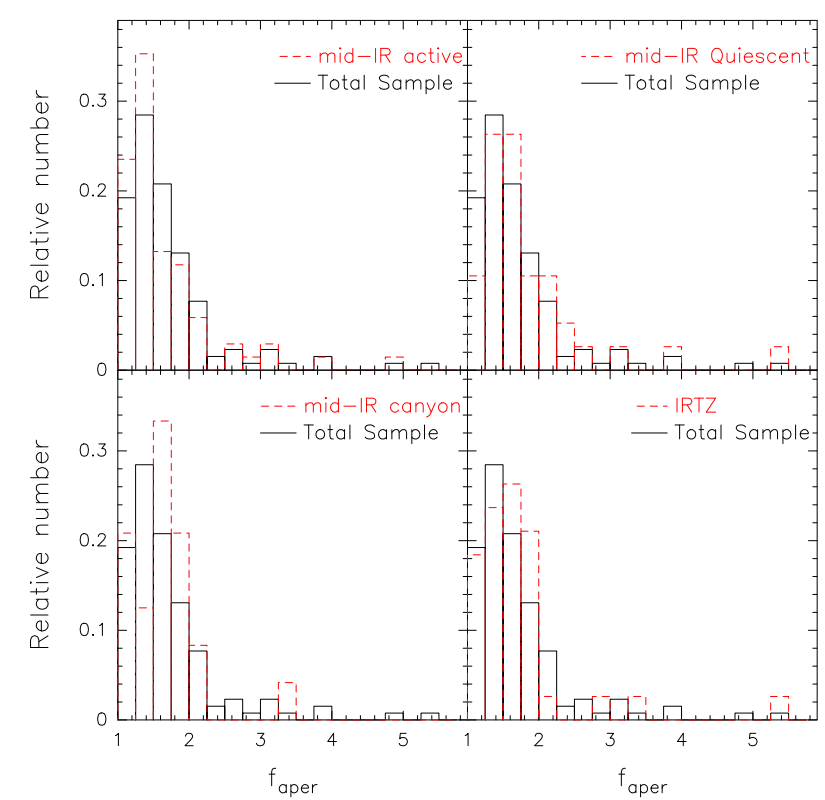

Fig. C.1. Histogram of the distribution of the aperture correction, $f_{\text {aper }}$, for the different subsamples (red dashed line) compared to the full sample (full black line).

Table C.2. Mean and median $\log \left(M_{\mathrm{mol}} / M_{*}\right)$ for galaxies with $f_{\text {aper }}<1.6$.

\begin{tabular}{|c|c|c|c|}
\hline Class & Mean & Median & $n / n_{\text {up }}{ }^{a}$ \\
\hline \multicolumn{4}{|c|}{ Galaxies with $f_{\text {aper }}<1.6$, all morphological types } \\
\hline Active & $-0.74 \pm 0.06$ & -0.85 & $48 / 14$ \\
\hline Canyon & $-1.20 \pm 0.06$ & -1.20 & $13 / 4$ \\
\hline IRTZ & $-1.39 \pm 0.13$ & -1.26 & $21 / 5$ \\
\hline quiescent & $-1.78 \pm 0.14$ & -1.82 & $19 / 10$ \\
\hline \multicolumn{4}{|c|}{ Galaxies with $f_{\text {aper }}<1.6$, late types $(T>0)$} \\
\hline Active & $-0.74 \pm 0.06$ & -0.76 & $39 / 11$ \\
\hline Canyon & $-1.10 \pm 0.07$ & -1.21 & $7 / 2$ \\
\hline IRTZ & $-1.20 \pm 0.13$ & -1.22 & 9/2 \\
\hline quiescent & $-1.41 \pm 0.13$ & -1.50 & $6 / 2$ \\
\hline
\end{tabular}

Notes. ${ }^{(a)}$ Total number of galaxies $(n)$ and number of upper limits $\left(n_{\mathrm{up}}\right)$.

Table C.3. Mean and median $\log \left(\mathrm{SFE}\right.$ ) (in units of $\mathrm{yr}^{-1}$ ) for galaxies with $f_{\text {aper }}<1.6$

\begin{tabular}{|c|c|c|c|}
\hline Class & Mean & Median & $n / n_{\text {up }}{ }^{a}$ \\
\hline \multicolumn{4}{|c|}{ Galaxies with $f_{\text {aper }}<1.6$, all morphological types } \\
\hline Active & $-9.17 \pm 0.06$ & -9.25 & $48 / 14$ \\
\hline Canyon & $-9.72 \pm 0.06$ & -9.70 & $13 / 4$ \\
\hline IRTZ & $-9.61 \pm 0.05$ & -9.62 & $21 / 5$ \\
\hline quiescent & $-9.63 \pm 0.09$ & -9.64 & $19 / 10$ \\
\hline \multicolumn{4}{|c|}{ Galaxies with $f_{\text {aper }}<1.6$, late types $(T>0)$} \\
\hline Active & $-9.23 \pm 0.06$ & -9.26 & $39 / 11$ \\
\hline Canyon & $-9.78 \pm 0.07$ & -9.86 & $7 / 2$ \\
\hline IRTZ & $-9.73 \pm 0.06$ & -9.78 & $9 / 2$ \\
\hline quiescent & $-9.79 \pm 0.09$ & -9.95 & $6 / 2$ \\
\hline
\end{tabular}

Notes. ${ }^{(a)}$ Total number of galaxies $(n)$ and number of upper limits $\left(n_{\text {up }}\right)$. 
A\&A 607, A110 (2017)

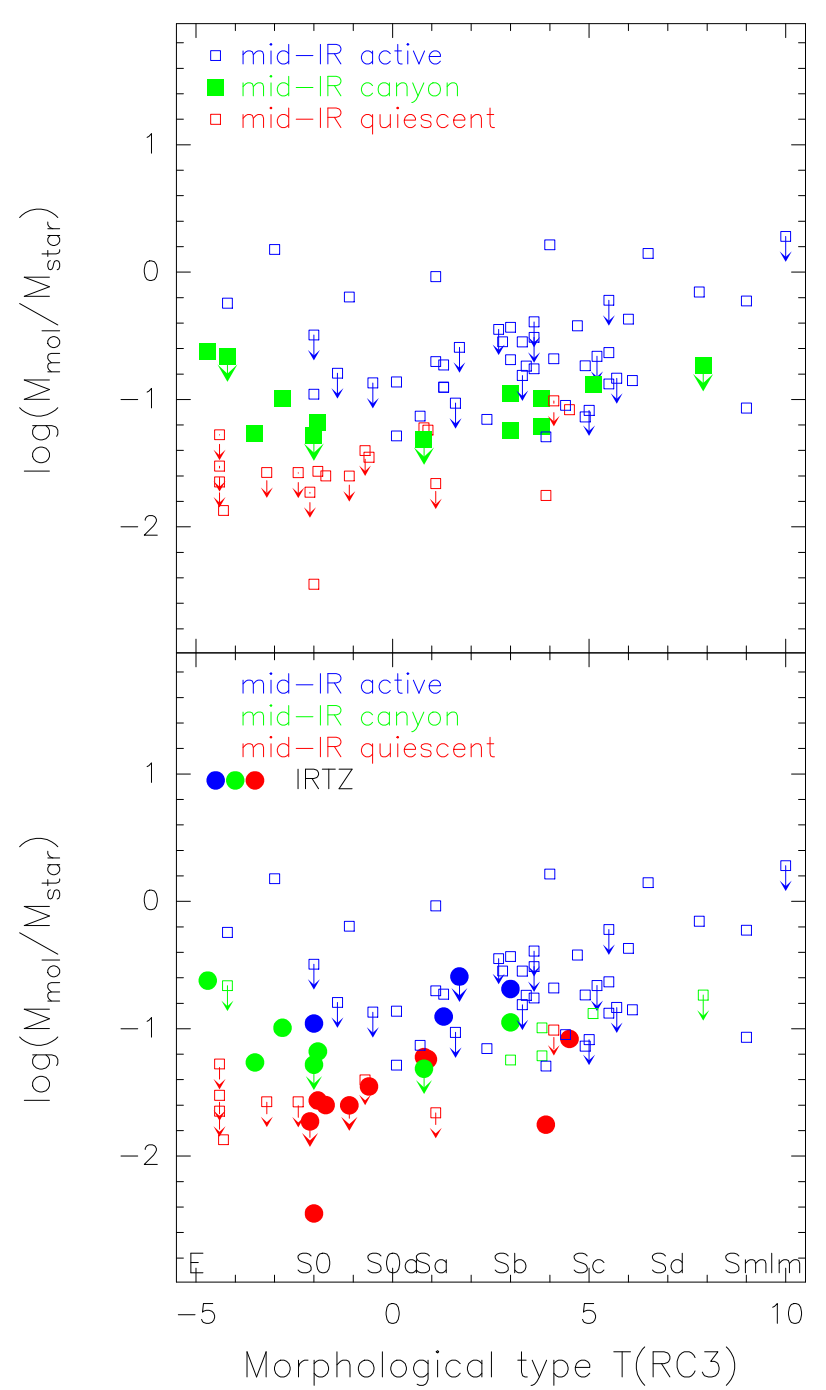

Fig. C.2. Ratio of molecular gas mass to stellar mass as a function of morphological type for galaxies with $f_{\text {aper }}<1.6$. The color coding is as in Fig. 1. Filled circles (lower panel) denote galaxies belonging to the IRTZ.

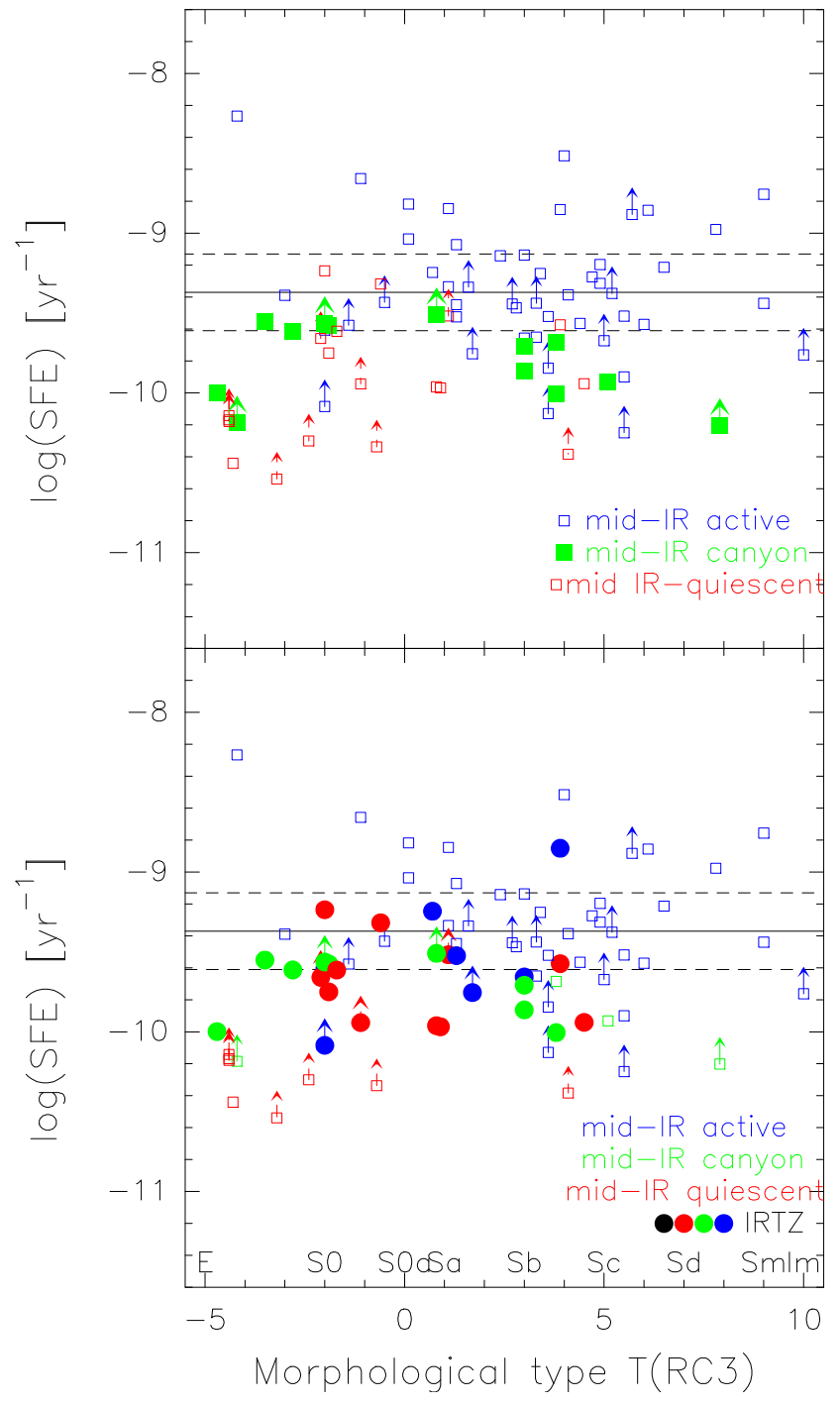

Fig. C.3. SFE as a function of morphological type for galaxies with $f_{\text {aper }}<1.6$. The objects are coded in color according to the classification of Zucker et al. (2016). The color coding is as in Fig. 1. The lines show the mean value (full line) and dispersion (dashed lines) found by Bigiel et al. (2011) for a sample of spiral and starburst galaxies. 